\title{
Dihedral f-Tilings of the Sphere by Equilateral and Scalene Triangles - III
}

\author{
A. M. d'Azevedo Breda* \\ Department of Mathematics \\ University of Aveiro \\ 3810-193 Aveiro, Portugal \\ ambreda@ua.pt \\ Patrícia S. Ribeiro* \\ Department of Mathematics \\ E.S.T. Setúbal \\ 2910-761 Setúbal, Portugal \\ pribeiro@est.ips.pt \\ Altino F. Santos ${ }^{\dagger}$ \\ Department of Mathematics \\ U.T.A.D. \\ 5001-801 Vila Real, Portugal \\ afolgado@utad.pt
}

Submitted: Oct 1, 2008; Accepted: Nov 26, 2008; Published: Dec 9, 2008

Mathematics Subject Classifications: 52C20, 52B05, 20B35

\begin{abstract}
The study of spherical dihedral f-tilings by equilateral and isosceles triangles was introduced in [3]. Taking as prototiles equilateral and scalene triangles, we are faced with three possible ways of adjacency. In [4] and [5] two of these possibilities were studied. Here, we complete this study, describing the f-tilings related to the remaining case of adjacency, including their symmetry groups. A table summarizing the results concerning all dihedral f-tilings by equilateral and scalene triangles is given in Table 2.
\end{abstract}

Keywords: dihedral f-tilings, combinatorial properties, symmetry groups

\footnotetext{
${ }^{*}$ Supported partially by the Research Unit Mathematics and Applications of University of Aveiro, through the Foundation for Science and Technology (FCT).

${ }^{\dagger}$ Research Unit CM-UTAD of University of Trás-os-Montes e Alto Douro.
} 


\section{Introduction}

Dihedral spherical folding tilings or dihedral f-tilings for short, are edge-to-edge decompositions of the sphere by geodesic polygons, such that all vertices are of even valency, the sums of alternate angles around each vertex are $\pi$ and every tile is congruent to one of two fixed sets $X$ and $Y$ (prototiles).

We shall denote by $\Omega(X, Y)$ the set, up to isomorphism, of all dihedral f-tilings of $S^{2}$ whose prototiles are $X$ and $Y$.

The classification of all dihedral spherical folding tilings by rhombi and triangles was obtained in 2005, [7]. However the analogous study considering two triangular (nonisomorphic) prototiles, $T_{1}$ and $T_{2}$ is not yet completed. This is not surprising, since it is much harder.

The case corresponding to prototiles given by an equilateral and an isosceles triangle was already described in [3].

When the prototiles are an equilateral and a scalene triangle, there are three distinct possibilities of adjacency, as shown in Figure 1.

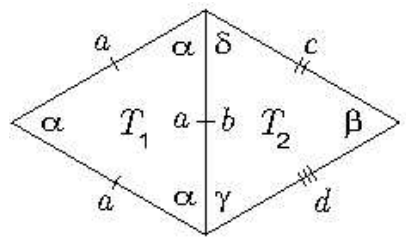

I

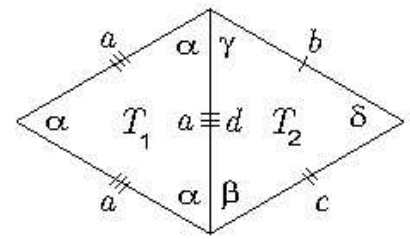

II

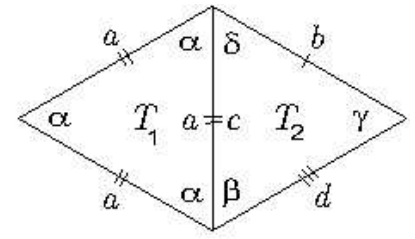

III

Figure 1: Distinct cases of adjacency.

We have already studied the cases corresponding to adjacency of Type I and II, see [4] and [5]. An interesting fact is that any tiling with adjacency of Type I or Type II can be seen as a subdivision of the sphere in $2 n, n \geq 2$ lunes with a pattern whose orbit under the action of a specific group covers the all sphere. Here, our interest is focused in spherical triangular dihedral f-tilings with adjacency of type III. As we shall see in this case we will find two families of tilings, $\mathcal{E}_{\alpha}$ and $\mathcal{G}^{k}$, with the same particularity, and four apparent sporadic tilings $(\mathcal{E}, \mathcal{F}, \mathcal{H}, \mathcal{L})$. However, these tilings can be seen, respectively, as new members of the following families (described in [5]) $\mathcal{F}_{p}$ and $\mathcal{D}_{p}$ allowing $p$ to be 3 , in both cases, and $\mathcal{E}^{m}$ allowing $m$ to be 3 or 4 .

From now on, $T_{1}$ denotes an equilateral spherical triangle of angle $\alpha\left(\alpha>\frac{\pi}{3}\right)$ and side $a$ and $T_{2}$ a scalene spherical triangle of angles $\delta, \gamma, \beta$, with the order relation $\delta<\gamma<$ $\beta(\delta+\gamma+\beta>\pi)$ and with sides $b$ (opposite to $\beta$ ), $c$ (opposite to $\gamma$ ) and $d$ (opposite to $\delta)$. The type III edge-adjacency condition can be analytically described by the equation

$$
\frac{\cos \alpha(1+\cos \alpha)}{\sin ^{2} \alpha}=\frac{\cos \gamma+\cos \delta \cos \beta}{\sin \delta \sin \beta}
$$

In order to get any dihedral f-tiling $\tau \in \Omega\left(T_{1}, T_{2}\right)$, we find it useful to start by considering one of its representations, beginning with a vertex common to an equilateral triangle 
and a scalene triangle in adjacent positions. In the diagrams that follows, it is convenient to label the tiles according to the following procedures:

(i) The tiles by which we begin the local configuration of a tiling $\tau \in \Omega\left(T_{1}, T_{2}\right)$ are labelled by 1 and 2, respectively;

(ii) For $j \geq 2$, the presence of a tile $j$ as shown can be deduced from the configuration of tiles $(1,2, \ldots, j-1)$ and from the hypothesis that the configuration is part of a complete local configuration of a f-tiling (except in the cases indicated).

\section{Triangular Dihedral F-Tilings with Adjacency of Type III}

Starting a local configuration of $\tau \in \Omega\left(T_{1}, T_{2}\right)$ with two adjacent cells congruent to $T_{1}$ and $T_{2}$ respectively (see Figure 2), a choice for angle $x \in\{\gamma, \beta\}$ must be made. We shall consider and study separately each one of the choices $\alpha+x=\pi$ and $\alpha+x<\pi, \quad x \in\{\gamma, \beta\}$.

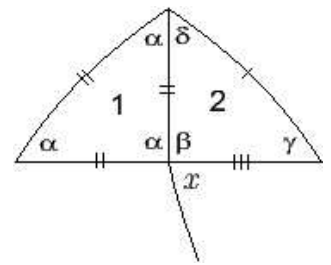

Figure 2: Local configuration.

With the above terminology one has:

Proposition 2.1. If $x=\gamma$ and $\alpha+x=\pi$, then $\Omega\left(T_{1}, T_{2}\right) \neq \emptyset$ if and only if $\beta+\delta=\pi$.

Proof. Suppose $x=\gamma$ and that $\alpha+x=\pi$. We may add some new cells to the configuration started in Figure 2 and get the one illustrated in Figure 3, with $\theta_{1} \in\{\beta, \gamma\}$.

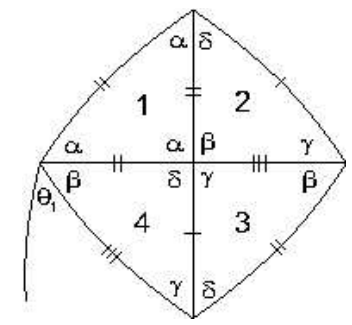

Figure 3: Local configuration.

If $\theta_{1}=\beta$, then $\alpha+\theta_{1} \leq \pi$, but since $\alpha+\gamma=\pi$ and $\gamma<\beta$, one has $\alpha+\theta_{1}>\pi$, which is a contradiction. 
If $\theta_{1}=\gamma$, we can expand the configuration in Figure 3 and obtain a global representation of a tiling $\tau_{\alpha} \in \Omega\left(T_{1}, T_{2}\right)$ as is shown in Figure 4. This family of tilings is composed by two equilateral and six scalene triangles and is denoted by $\mathcal{E}_{\alpha}$.
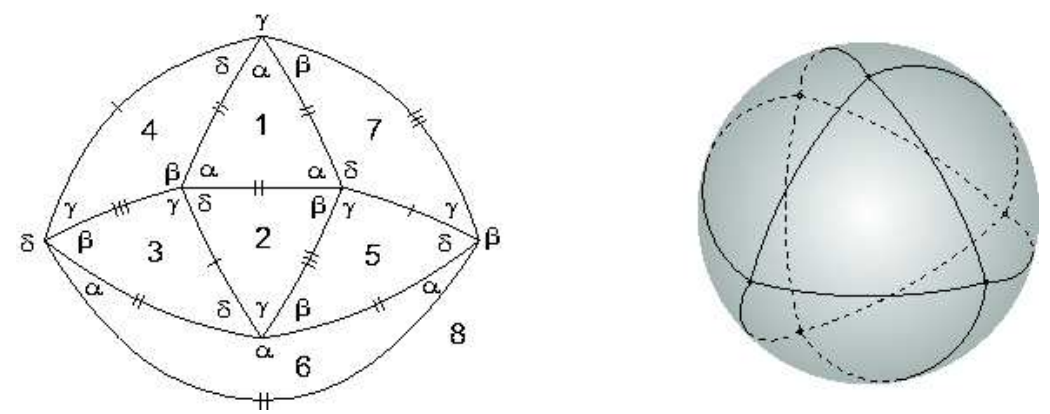

Figure 4: 2D and 3D representation of $\mathcal{E}_{\alpha}$.

By the adjacency condition (1.1), the condition $\alpha+\gamma=\pi=\beta+\delta$ and the order relation between the angles, we may conclude that $\beta>\alpha>\frac{\pi}{2}$.

Proposition 2.2. If $x=\gamma$ and $\alpha+x<\pi$, then $\Omega\left(T_{1}, T_{2}\right) \neq \emptyset$ if and only if $\alpha+\gamma+k \delta=$ $\pi, \beta+\gamma=\pi$ and $\beta+(k+1) \delta=\pi$, for some $k \geq 1$. In this situation, for each $k \geq 1$, there is a single f-tiling denoted by $\mathcal{G}^{k}$.

Proof. Suppose that $\alpha+x<\pi$, with $x=\gamma$ (see Figure 2). We are led to the configuration illustrated in Figure 5 and a decision must be taken about the angle labelled $\theta_{2} \in\{\gamma, \delta\}$ :

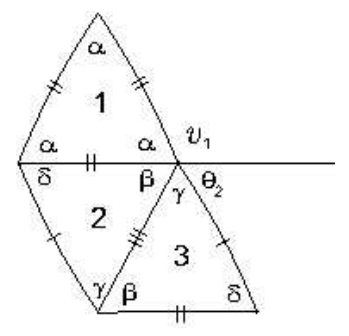

Figure 5: Local configuration.

1. If $\theta_{2}=\gamma$, then $\beta+\theta_{2}<\pi$ and since $\gamma<\beta$, we get $\delta<\gamma<\frac{\pi}{2}$. Consequently $\alpha \geq \frac{\pi}{2}$ or $\beta \geq \frac{\pi}{2}$, since vertices of valency four must exist (see [6]).

1.1 If $\alpha \geq \frac{\pi}{2}$, from the adjacency condition (1.1), $\beta>\frac{\pi}{2}$ and so the sum $\beta+\theta_{2}+\lambda$ does not satisfy the angle folding relation for each $\lambda \in\{\alpha, \delta, \gamma, \beta\}$.

1.2 If $\beta \geq \frac{\pi}{2}$, the configuration in Figure 5 ends up in a contradiction since, in order to satisfy the angle folding relation, the sum of alternate angles containing $\beta$ and $\theta_{2}=\gamma$ 
must be $\beta+\gamma+\alpha=\pi$ and the other sum is $\alpha+2 \gamma=\pi$ leading to $\gamma=\beta$, which is impossible.

2. Suppose now that $\theta_{2}=\delta$. As $\alpha+\gamma<\pi$, then $\beta+\theta_{2}<\pi$ and consequently $\delta<\frac{\pi}{2}$. Additionally, $\gamma<\frac{\pi}{2}$, otherwise $\beta>\gamma \geq \frac{\pi}{2}, \alpha \leq \frac{\pi}{2}$ and the adjacency condition (1.1) is not fulfilled. Accordingly, $\delta<\gamma<\frac{\pi}{2}$ and vertices of valency four occur if and only if $\alpha \geq \frac{\pi}{2}$ or $\beta \geq \frac{\pi}{2}$.

2.1 If $\alpha=\frac{\pi}{2}$, by the adjacency condition (1.1), $\beta>\frac{\pi}{2}$. We may add some new cells to the configuration shown in Figure 5, obtaining the following one:

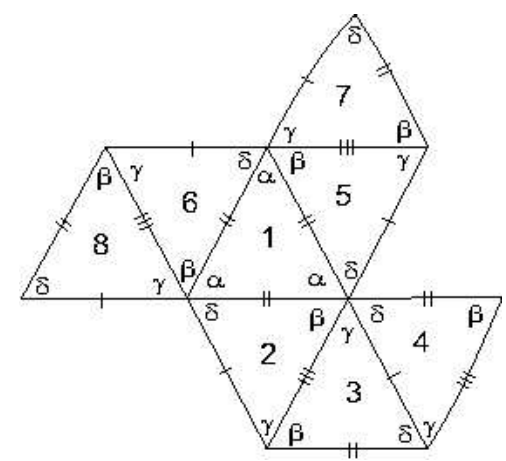

Figure 6: Local configuration.

The sum containing alternate angles $\beta$ and $\delta$ must satisfy $\beta+k \delta=\pi$, for some $k>1$ and taking into account the edge compatibility, we conclude that the other sum is $\alpha+\gamma+(k-1) \delta=\pi$. Therefore, $\beta+\delta=\frac{\pi}{2}+\gamma$ and by the adjacency condition (1.1),

$$
\begin{array}{r}
\cos \gamma=-\cos \beta \cos \delta \Leftrightarrow \sin (\beta+\delta)=-\cos \beta \cos \delta \\
\Leftrightarrow \sin (\pi-k \delta+\delta)=\cos (k \delta) \cos \delta \\
\Leftrightarrow-\sin (k \delta-\delta)=\cos (k \delta) \cos \delta
\end{array}
$$

Taking into account that $k \delta<\frac{\pi}{2}$, then $\sin (k \delta-\delta)<0$ and so $k \delta-\delta>\pi$, which is an impossibility.

2.2 If $\alpha>\frac{\pi}{2}$, from the adjacency condition (1.1), we conclude that $\delta<\gamma<\frac{\pi}{2}<\beta$. Since $\alpha+\gamma<\pi, \quad \alpha+\delta<\pi$ and $\beta+\delta<\pi$, vertices of valency four are surrounded by alternate angles $\beta$ and $\gamma$, which violates the adjacency condition.

2.3 If $\beta=\frac{\pi}{2}$, then $\alpha<\frac{\pi}{2}$ and vertices of valency four are surrounded exclusively by angles $\beta$.

Since $\gamma+\delta>\frac{\pi}{2}$ and $\gamma>\frac{\pi}{4}$, the angular sum containing $\alpha$ and $\gamma$ must be $2 \alpha+\gamma=$ $\pi, \alpha+2 \gamma=\pi$ or $\alpha+\gamma+p \delta=\pi$, for some $p \geq 1$. We shall study each case separately. 
2.3.1 The vertices of valency six in which one of the sums of alternate angles is $2 \alpha+\gamma=\pi$ are surrounded by the angular sequence $(\alpha, \alpha, \alpha, \beta, \gamma, \delta)$. By the adjacency condition, we conclude that $\alpha=\frac{\pi}{3}$ or approximately $128,17^{\circ}$, which is impossible in both cases.

2.3.2 In case $\alpha+2 \gamma=\pi$, the angle arrangement around vertex $v_{1}$, in Figure 5 (valency six) is impossible since $\theta_{2}=\delta$.

2.3.3 Assume now that $\alpha+\gamma+p \delta=\pi$, for some $p \geq 1$. Extending the configuration in Figure 5, we get the one below:

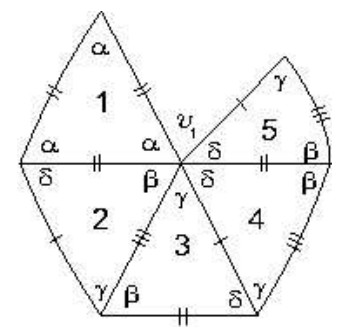

Figure 7: Local configuration.

The sum of the alternate angles, at vertex $v_{1}$, containing $\beta$ and $\delta$ must satisfy $\beta+t \delta=\pi$, for some $t>1$. Then, $\beta+t \delta=\pi=\alpha+\gamma+(t-1) \delta=\pi$ and so $\beta+\delta=\alpha+\gamma$. Consequently, $\delta>\frac{\pi}{12}$ and $\delta=\frac{\pi}{2 t}, \quad t=2,3,4,5$. By the adjacency condition (1.1), one has

$$
-\cos (\gamma+(t-1) \delta) \sin \delta=\cos \gamma(1+\cos (\gamma+(t-1) \delta))
$$

and for $t=2,3,4,5$ we get, respectively, $\gamma \approx 66.26^{\circ}, \gamma=\frac{\pi}{3}, \gamma \approx 57,98^{\circ}, \gamma \approx 57.44^{\circ}$ and $\alpha \approx 68.74^{\circ}, \alpha=\frac{\pi}{3}, \alpha \approx 54.52^{\circ}, \alpha \approx 50.56^{\circ}$. Taking into account that $\alpha>\frac{\pi}{3}$, then $t=2$. However, extending the configuration in Figure 7, we get a vertex surrounded by three consecutive angles $\gamma$, whose sum $2 \gamma+\mu$ violates the angle folding relation, where $\mu$ denotes a sum of angles containing $\alpha, \delta, \gamma$ or $\beta$ (see Figure 8 ).

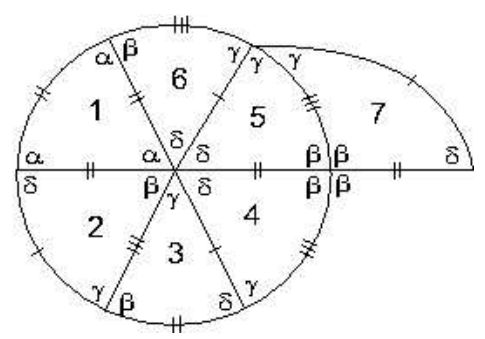

Figure 8: Local configuration.

2.4 Consider $\beta>\frac{\pi}{2}$. If $\alpha>\frac{\pi}{2}$, the vertices of valency four are surrounded by alternate angles $\beta$ and $\gamma$. But, since $\beta+\delta<\pi, \alpha+\delta<\alpha+\gamma<\pi$, the sum $\beta+\gamma=\pi$ violates the adjacency condition (1.1) and so $\alpha \leq \frac{\pi}{2}$. 
2.4.1 If $\alpha=\frac{\pi}{2}$, then $\beta+\gamma \neq \pi$, otherwise, by the adjacency condition (1.1) $\delta=0$. The configuration started in Figure 5, with $\theta_{2}=\delta$, extends to the one shown in the next figure.

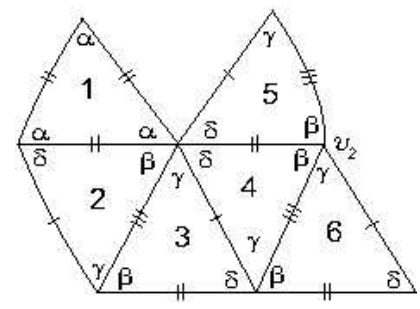

Figure 9: Local configuration.

Looking at vertex labelled $v_{2}$, we observe that the sum containing the alternate angles $\beta$ and $\gamma$ is of the form $\beta+\gamma+\lambda$, which does not satisfy the angle folding relation for any $\lambda \in\{\alpha, \beta, \gamma\}$.

2.4.2 Assume now that $\alpha<\frac{\pi}{2}$. Adding a new cell in the configuration of Figure 5, a decision must be taken about the angle $\theta_{3} \in\{\alpha, \delta, \beta\}$ as is illustrated in Figure 10:

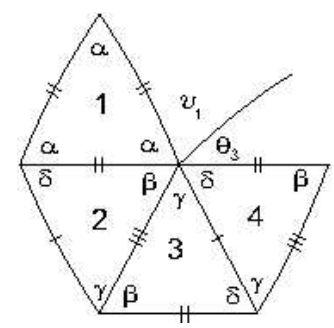

Figure 10: Local configuration.

2.4.2.1 Suppose $\theta_{3}=\alpha$. Then, $2 \alpha+\gamma \leq \pi$ and consequently $\gamma<\frac{\pi}{3}$. If $2 \alpha+\gamma=\pi$, then the other sum of alternate angles at vertex $v_{1}$ must be $\beta+\delta+\alpha=\pi$ and so $\alpha+\gamma=\beta+\delta$. Taking into account that $\beta+\gamma+\delta>\pi$, we conclude that $2 \gamma+\alpha>\pi$ and consequently $\gamma>\alpha>\frac{\pi}{3}$, contradicting $\gamma<\frac{\pi}{3}$.

If $2 \alpha+\gamma<\pi$, we can add some cells to the configuration illustrated in Figure 10 and obtain the one in Figure 11.

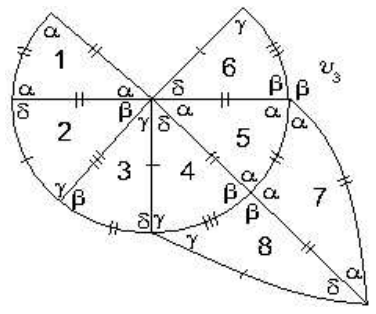

Figure 11: Local configuration. 
Observe that if tile 6 is an equilateral triangle, the sum $\alpha+\delta+\beta$ implies that vertices of valency four must be surrounded by alternate angles $\beta$ and $\gamma$. Consequently $\beta>\frac{2 \pi}{3}$, contradicting $\beta+\delta+\alpha \leq \pi$. Still, note that in the construction of the configuration, vertex $v_{3}$ is of valency four, otherwise these types of vertices would be surrounded by alternate angles $\beta$ and $\gamma$ leading to the same contradiction above.

Since $\alpha+\beta=\pi$ and $\beta+\gamma+\delta>\pi$, one has $\gamma+\delta>\alpha>\frac{\pi}{3}$ and $\gamma>\frac{\pi}{6}$. Then, $2 \alpha+\gamma+\lambda>\pi$, for any $\lambda \in\{\alpha, \delta, \gamma, \beta\}$, which is an impossibility.

2.4.2.2 Suppose now that $\theta_{3}=\delta$. Then, $\alpha+\gamma+\delta \leq \pi$. If $\alpha+\gamma+\delta=\pi$, the configuration in Figure 10 ends up to the one illustrated in Figure 12.

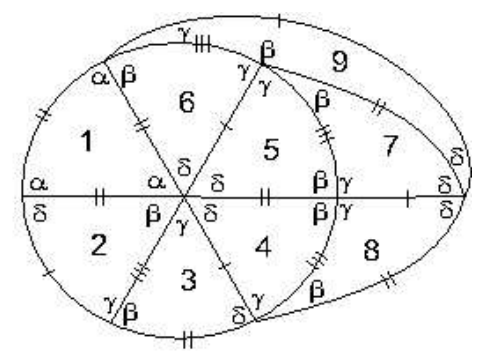

Figure 12: Local configuration.

From the adjacency condition $(1.1), \delta \approx 32.31^{\circ}, \gamma \approx 64.63^{\circ}, \beta \approx 115.38^{\circ}$ and $\alpha \approx 83.07^{\circ}$ and the configuration extends to a tiling $\tau \in \Omega\left(T_{1}, T_{2}\right)$. It is composed of two equilateral and eighteen scalene triangles and will be denoted by $\mathcal{G}^{1}$, Figure 13.
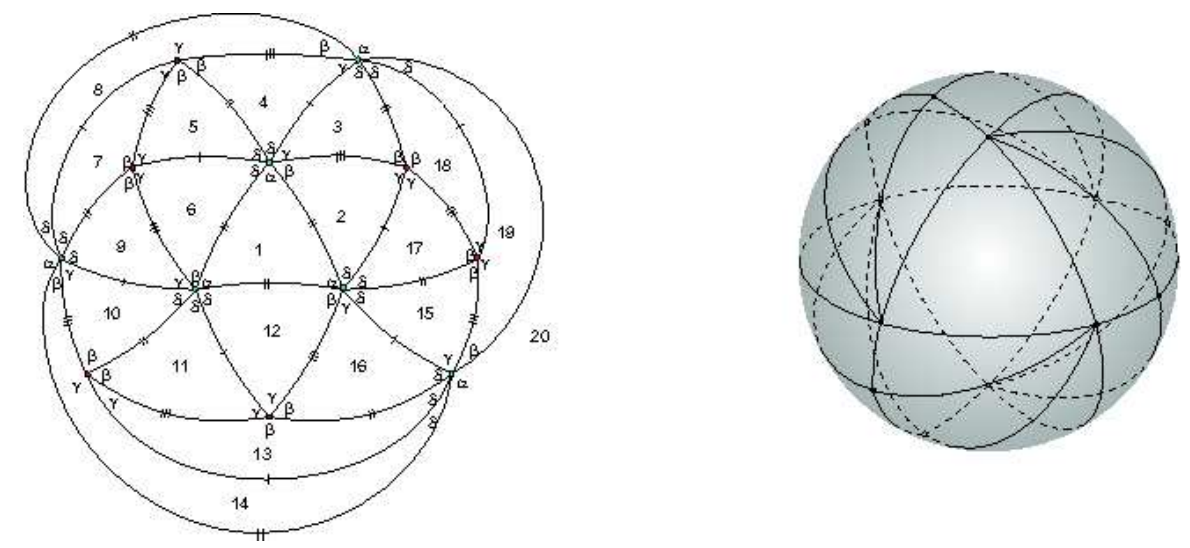

Figure 13: 2D and 3D representation of $\mathcal{G}^{1}$.

Assume now that $\alpha+\gamma+\delta<\pi$ (see Figure 10). Adding new cells to the configuration we conclude that $\beta+\gamma \leq \pi$, Figure 14. In case $\beta+\gamma<\pi$, then $\beta+\alpha=\pi$, since vertices of valency four must exist. Taking into account that $\beta+\gamma+\delta>\pi$, we conclude that $\gamma>\frac{\pi}{6}$ and consequently $\beta+\gamma+\lambda>\pi$, for each $\lambda \in\{\alpha, \gamma, \beta, \delta\}$. Therefore, the configuration cannot be expanded. 


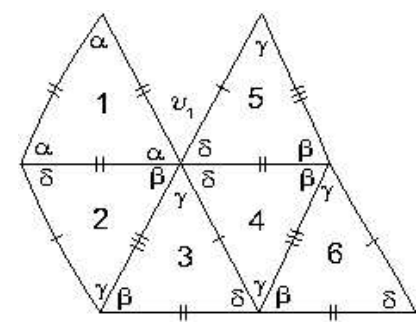

Figure 14: Local configuration.

At vertex $v_{1}$, the sum of alternate angles containing $\beta$ and $\delta$ satisfies $\beta+k \delta=\pi$ or $\beta+\alpha+t \delta=\pi$, for $k \geq 2$ and $t \geq 1$.

2.4.2.2.1 Assuming that $\beta+k \delta=\pi, k \geq 2$, then the other sum of angles at the same vertex satisfies $\alpha+\gamma+(k-1) \delta=\pi$, as is shown in Figure 15 .

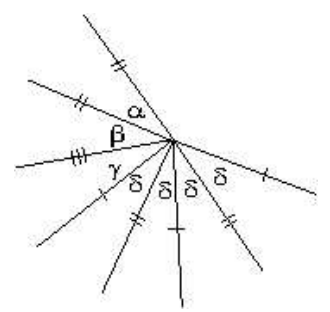

Figure 15: Angle arrangement around vertices surrounded by alternate $\beta$ and $\delta$.

We may now expand the configuration in Figure 10 getting a tiling $\tau \in \Omega\left(T_{1}, T_{2}\right)$. In Figure 16 we present a 2D and 3D representation of this tiling with $k=2$, which is denoted by $\mathcal{G}^{2}$. The corresponding f-tiling is composed by two equilateral triangles and thirty scalene triangles, $\delta \approx 19.08^{\circ}, \gamma \approx 57.24^{\circ}, \beta \approx 122.76^{\circ}$ and $\alpha \approx 84.60^{\circ}$. Generalizing, for $k \geq 1$, the corresponding f-tiling, $\mathcal{G}^{k}$ is composed by two equilateral triangles and $6(2 k+1)$ scalene triangles.
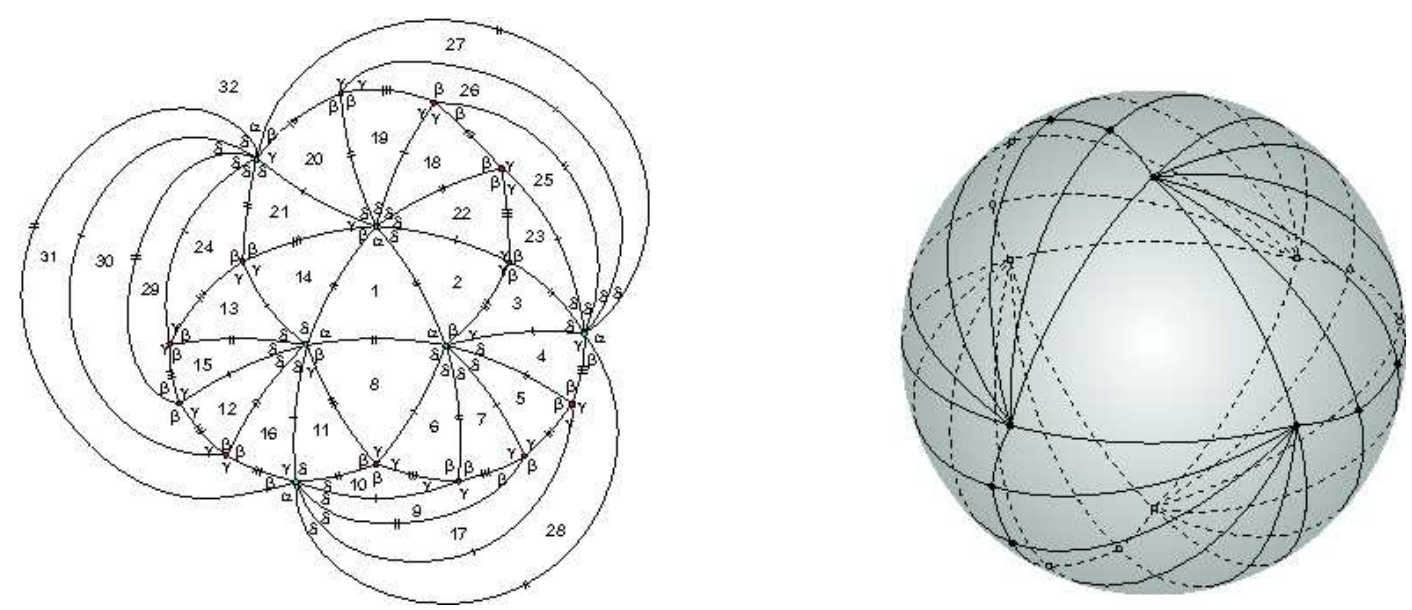

Figure 16: 2D and 3D representation of $\mathcal{G}^{2}$. 
If the restriction of edge-to edge tiling was removed it would not be difficult to construct new tilings, starting from $\mathcal{G}^{k}$, with a similar pattern as the Dawson's swirl tiling illustrated in Figure 10 of [8].

2.4.2.2.2 If $\beta+\alpha+t \delta=\pi$, then $t \geq 2$, otherwise $\beta=\gamma$. Taking into account that $\beta+\gamma=\pi$, we get $\gamma>\alpha>\frac{\pi}{3}$ and so the vertices surrounded by the alternate angles $\alpha, \gamma$ and $\delta$ satisfy $\alpha+\gamma+t \delta=\pi$. Consequently, at vertex $v_{1}$, both sums of the alternate angles are of the form $\alpha+\gamma+t \delta=\pi=\beta+\alpha+t \delta$, which is an impossibility, since $\gamma<\beta$.

2.4.2.3 Suppose finally that $\theta_{3}=\beta$ (see Figure 10). Since vertices of valency four must be surrounded by alternate angles $\beta$ and $\alpha$ or $\beta$ and $\gamma$, then the sequence of alternate angles around vertex $v_{1}$ is impossible.

Proposition 2.3. If $x=\beta$ and $\alpha+x=\pi$, then $\Omega\left(T_{1}, T_{2}\right)$ is composed of four isolated dihedral triangles f-tilings $\mathcal{E}, \mathcal{F}, \mathcal{H}$ and $\mathcal{L}$, such that the sum of alternate angles around vertices are respectively of the form:

$$
\begin{array}{r}
\alpha+\beta=\pi, \alpha+2 \delta=\pi \text { and } \gamma=\frac{\pi}{3}, \text { for } \mathcal{E} ; \\
\alpha+\beta=\pi, 2 \alpha+\delta=\pi \text { and } \gamma=\frac{\pi}{3}, \text { for } \mathcal{F} ; \\
\alpha+\beta=\pi, \alpha+2 \delta+\gamma=\pi \text { and } \gamma=\frac{\pi}{3}, \text { for } \mathcal{H} ; \\
\alpha+\beta=\pi, \alpha+2 \delta+\gamma=\pi \text { and } \gamma=\frac{\pi}{4}, \text { for } \mathcal{L} .
\end{array}
$$

Proof. Let us assume that $x=\beta$ and $\alpha+x=\pi$ in Figure 2. Then, $\gamma+\delta>\alpha>\frac{\pi}{3}$ and $\gamma>\frac{\pi}{6}$. The configuration started in Figure 2 extends to the one illustrated in Figure 17 .

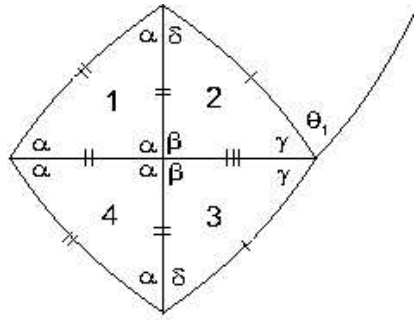

Figure 17: Local configuration.

A decision must be taken about the angle labelled $\theta_{1} \in\{\gamma, \delta\}$.

1. Assuming that $\theta_{1}=\gamma$, then $\gamma \leq \frac{\pi}{2}$. If $\gamma=\frac{\pi}{2}$, then $\beta>\frac{\pi}{2}, \quad \delta<\frac{\pi}{2}$ and $\alpha<\frac{\pi}{2}$, which is impossible by the adjacency condition (1.1).

Therefore, $\delta<\gamma<\frac{\pi}{2}$ and again, by the adjacency condition, we conclude that $\alpha<\frac{\pi}{2}<\beta$. Since we are assuming that $\theta_{1}=\gamma$, the configuration extends a bit more to the one shown in Figure 18 and angle $\theta_{2}$ must be $\gamma$, otherwise the sum containing $\theta_{2}=\beta$ and $\gamma$ would be simply $\beta+\gamma$ or $\beta+\gamma+\lambda$. 
In the first case, the other sum of angles would satisfy $2 \gamma=\pi$, which is impossible and in the second case the angle folding relation is violated, for any $\lambda \in\{\alpha, \delta, \gamma, \beta\}$.

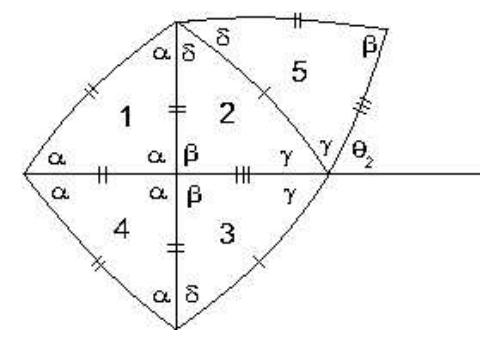

Figure 18: Local configuration.

Adding one new cell to the configuration in Figure 18, we get the following one:

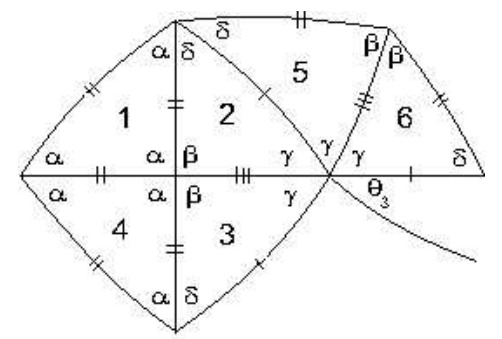

Figure 19: Local configuration.

1.1 Suppose firstly, that $\theta_{3}=\gamma$ and $\gamma=\frac{\pi}{3}$. We may extend the configuration in Figure 19 and a decision must be taken about the angle $\theta_{4} \in\{\beta, \alpha\}$, as is shown in Figure 20.

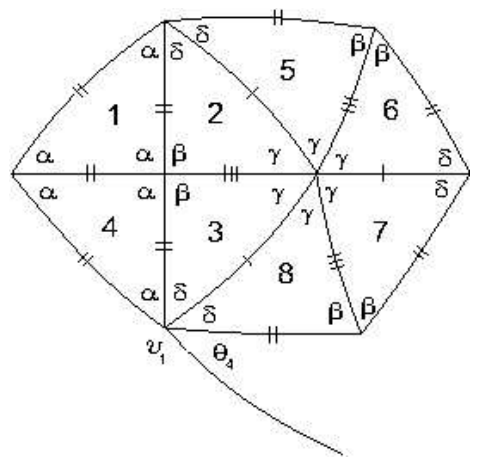

Figure 20: Local configuration.

1.1.1 If $\theta_{4}=\beta$, then the sum of the alternate angles containing $\theta_{4}=\beta$ and $\delta$ at vertex $v_{1}$ satisfies $\beta+t \delta=\pi$ and the other $\alpha+t \delta=\pi$ or $\alpha+\gamma+(t-1) \delta=\pi$ or $2 \alpha+(t-1) \delta=\pi$, for some $t \geq 2$.

In the first case, we get $\alpha=\beta$, which is impossible. In the second case, by the adjacency condition (1.1), we conclude that 


$$
\frac{\cos \alpha(1+\cos \alpha)}{\sin ^{2} \alpha}=\frac{\frac{1}{2}+\cos \alpha \cos \left(2 \alpha+\frac{\pi}{3}\right)}{-\sin \alpha \sin \left(2 \alpha+\frac{\pi}{3}\right)} .
$$

Since $\frac{\pi}{3}<\alpha<\frac{\pi}{2}$, then $\alpha \approx 69.12^{\circ}, \beta \approx 110.84^{\circ}$ and $\delta \approx 18.31^{\circ}$, which is impossible for any $t \geq 2$.

In the third case, by the adjacency condition (1.1),

$$
\frac{\cos \alpha(1+\cos \alpha)}{\sin ^{2} \alpha}=\frac{\frac{1}{2}+\cos \alpha \cos (3 \alpha)}{-\sin \alpha \sin (3 \alpha)}
$$

and so $\alpha=\frac{2 \pi}{5}, \beta=\frac{3 \pi}{5}$ and $\delta=\frac{\pi}{5}$. Therefore, $t=2$, but by edge compatibility, we conclude that it is impossible to pursuing the configuration.

1.1.2 Suppose $\theta_{4}=\alpha$. The sum of the alternate angles containing $\theta_{4}=\alpha$ and $\delta$ satisfies $\alpha+t \delta=\pi$, for some $t \geq 2$ or $2 \alpha+p \delta=\pi$, for some $p \geq 1$ or $\alpha+\gamma+q \delta=\pi$, for some $q \geq 1$.

1.1.2.1 In the first case, we have $\alpha=\pi-t \delta, \beta=t \delta$ and $\gamma=\frac{\pi}{3}$. For $t=2$ the configuration extends globally to the one illustrated in Figure 21 and is denoted by $\mathcal{E}$.
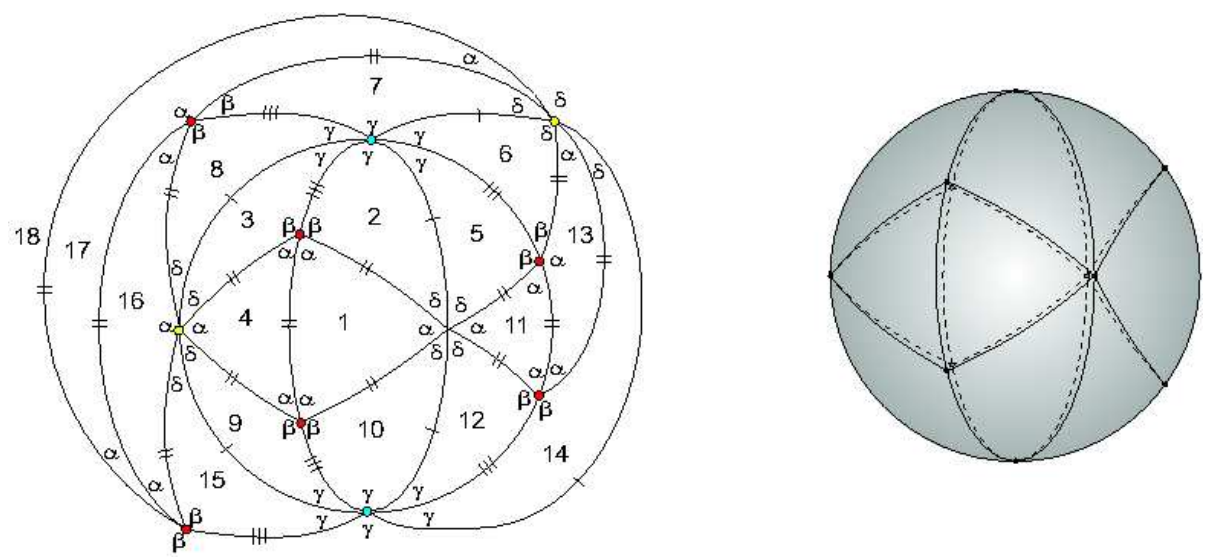

Figure 21: 2D and 3D representation of $\mathcal{E}$.

This tiling has six equilateral triangles and twelve scalene triangles and it was expanded in an unique way. By the adjacency condition (1.1), we conclude that $\alpha \approx 72,75^{\circ}, \beta \approx$ $107,25^{\circ}$ and $\delta \approx 53,63^{\circ}$. 
For $t>2$, the local representation ends up at a vertex $v_{2}$ surrounded by angles $\beta, \beta, \gamma$, whose sum $\beta+\gamma$ does not satisfy the angle folding relation.

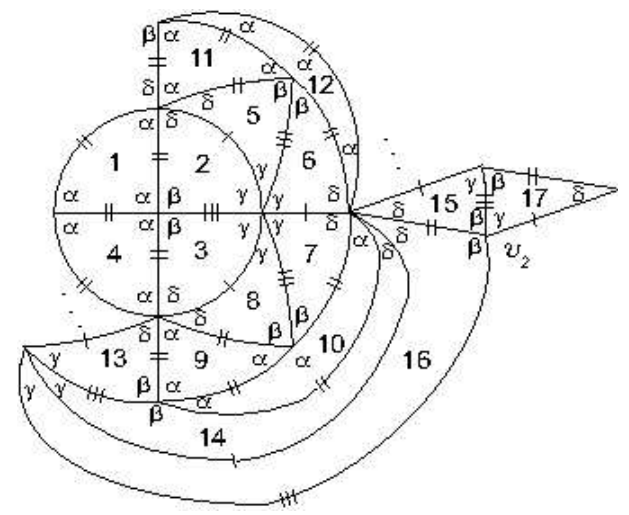

Figure 22: Local configuration.

1.1.2.2 In the second case, we have $2 \alpha+p \delta=\pi$ and for $p=1$, we get a global representation of a tiling $\tau \in \Omega\left(T_{1}, T_{2}\right)$, where $\alpha=\frac{2 \pi}{5}, \delta=\frac{\pi}{5}$ and $\beta=\frac{3 \pi}{5}$. It has twelve equilateral triangles and twelve scalene triangles and is denoted by $\mathcal{F}$. In Figure 23 we present a 2D and 3D representation of this f-tiling and this construction corresponds to a choice of the sides of tile 15 , in order to avoid vertices surrounded by the angular sequence $(\alpha, \alpha, \alpha, \beta, \delta, \ldots)$, which does not satisfy the angle folding relation.
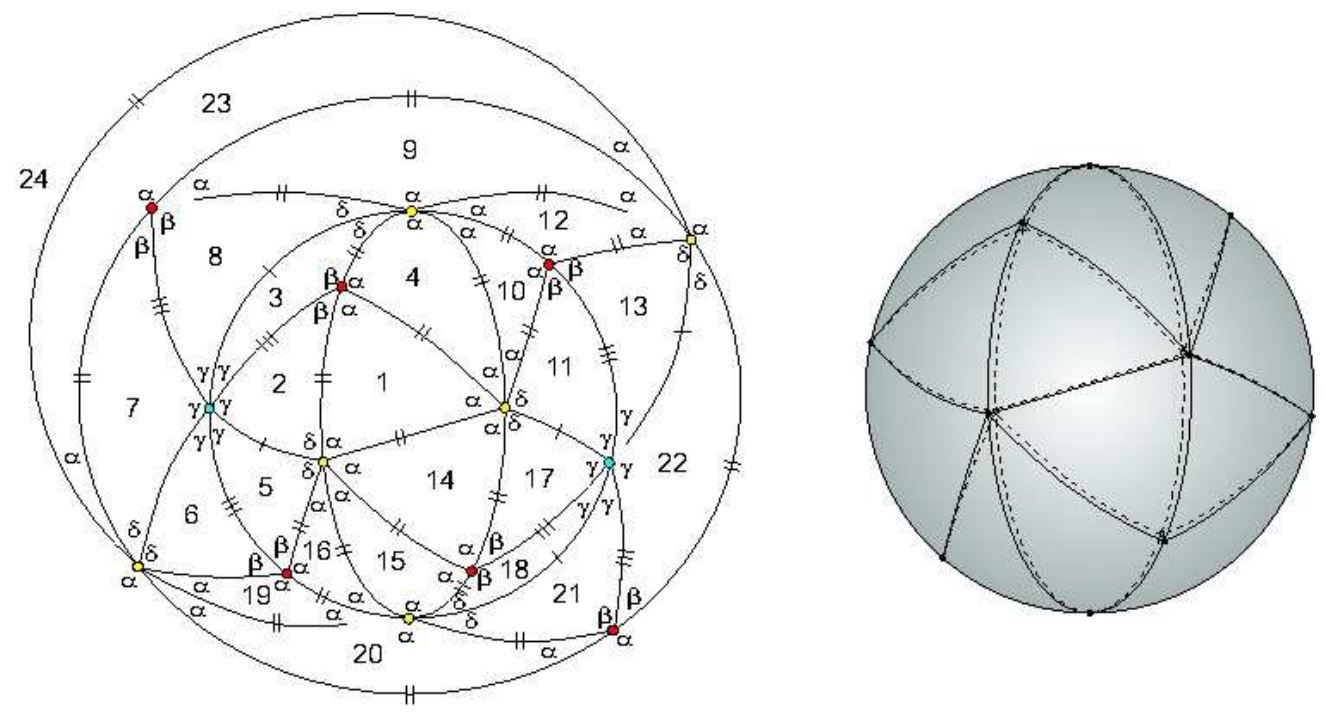

Figure 23: 2D and 3D representation of $\mathcal{F}$.

For $p>1$ and assuming that tile 10 is an equilateral triangle in the positions illustrated below, we always get vertices surrounded by alternate angles $\beta$ and $\gamma$ (see Figure 24-I, II and III), whose sum does not satisfy the angle folding relation. Note that to avoid vertices surrounded by angles $\beta, \alpha, \beta$ (whose sum $2 \beta$ does not satisfy the angle folding 
relation), tile 17 in 24-I must be an equilateral triangle and to avoid vertices surrounded by angles $(\beta, \alpha, \delta, \delta, \ldots)$ (which is incompatible with the edge sides), tiles 15 in 24-II and 18 in 24-III must be the ones illustrated.

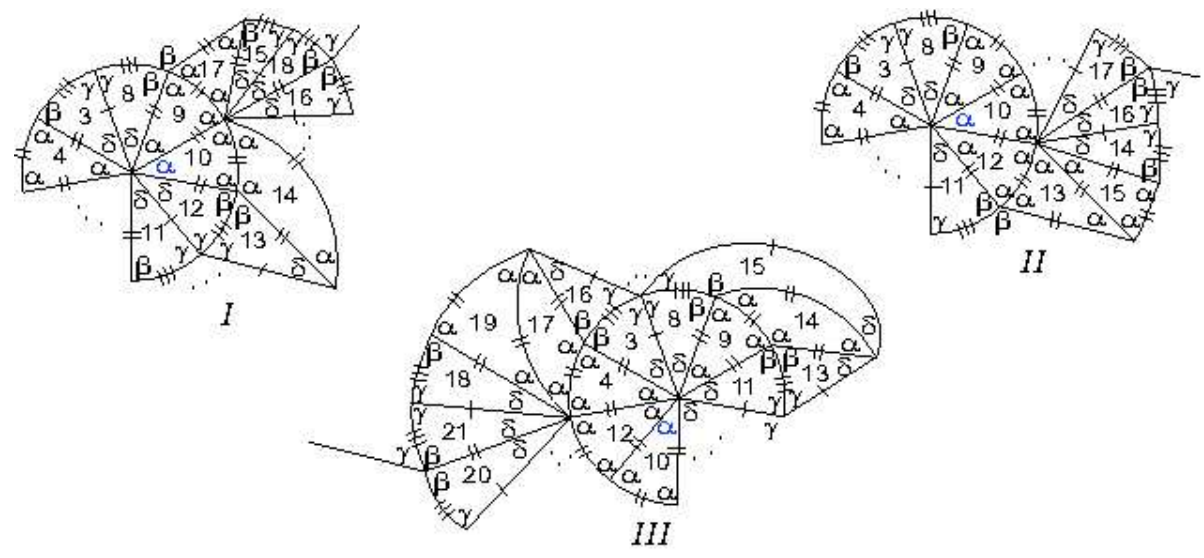

Figure 24: Local configuration.

The other position for the equilateral triangle in tile 10 is shown in Figure 25 and once again, we end up at a vertex surrounded by angles $\beta, \beta, \gamma$ or $\beta, \gamma, \gamma$, whose sum $\beta+\gamma$ does not satisfy the angle folding relation.
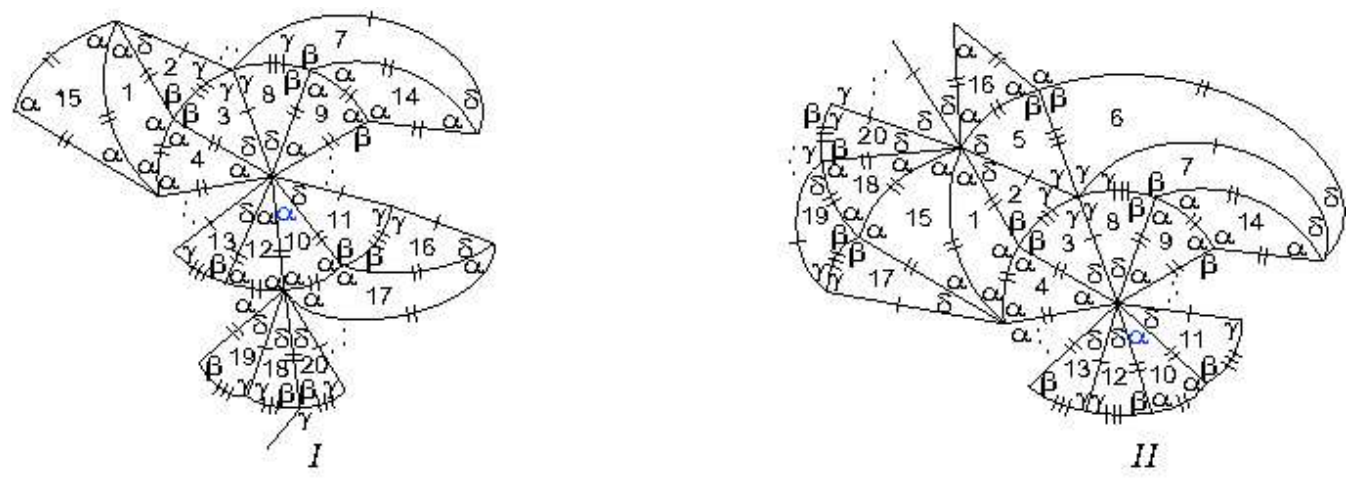

Figure 25: Local configuration.

1.1.2.3 In the third case, $\alpha+\gamma+q \delta=\pi$, for $q \geq 1$ and if $q=1$, we get an impossibility due to the edge compatibility of the triangles. For $q=2$, we get $\alpha \approx 70.52^{\circ}, \delta \approx 24.74^{\circ}$ and $\beta \approx 109.48^{\circ}$ and we may expand globally the configuration obtaining a representation of a tiling $\tau \in \Omega\left(T_{1}, T_{2}\right)$, which is denoted by $\mathcal{H}$, see Figure 26. It is composed of twelve equilateral triangles and twenty four scalene triangles. 

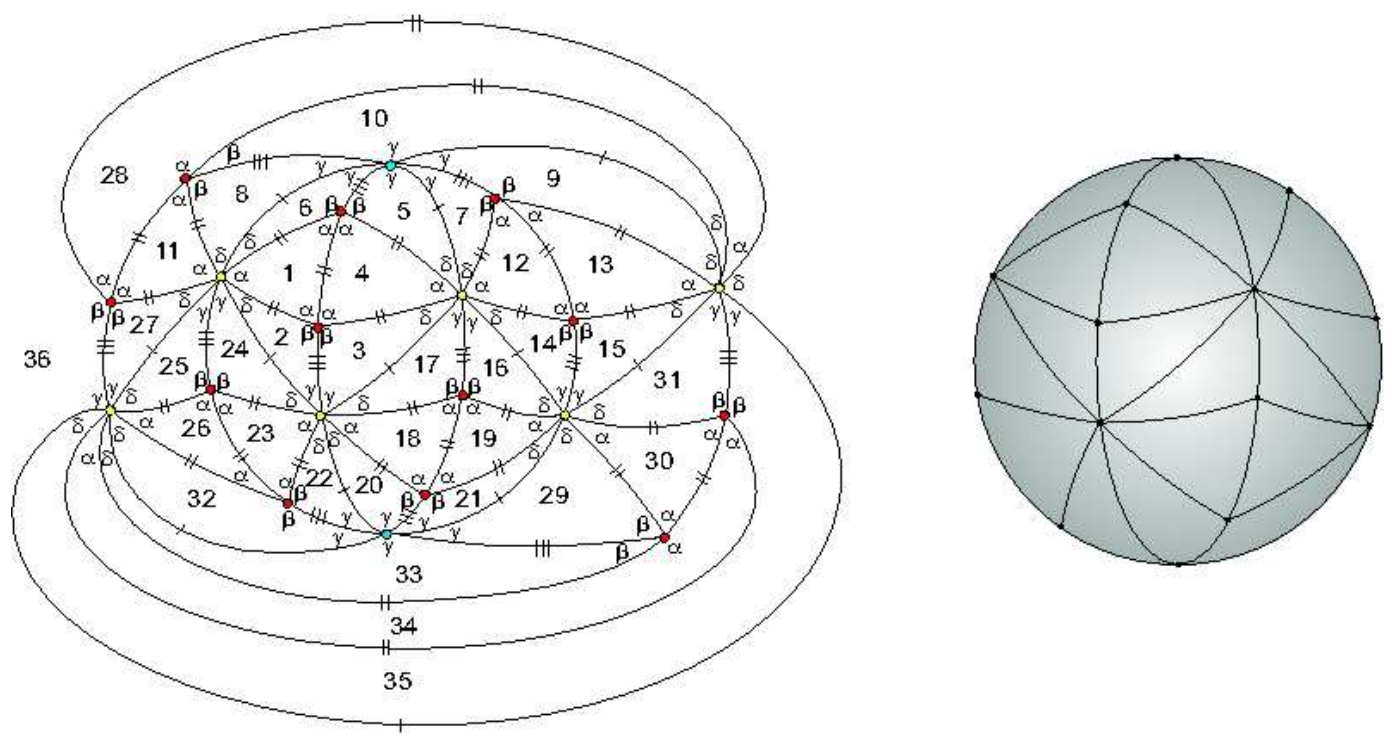

Figure 26: 2D and 3D representation of $\mathcal{H}$.

For $q>2$, we observe that the angle arrangement at vertices whose sum of alternate angles satisfy $\alpha+\gamma+q \delta=\pi$ has always three consecutive angles $\delta$ leading to a vertex surrounded by angles $\beta, \beta, \gamma$, as is illustrated in Figure 27 for cases $q=3$ and $q=4$.
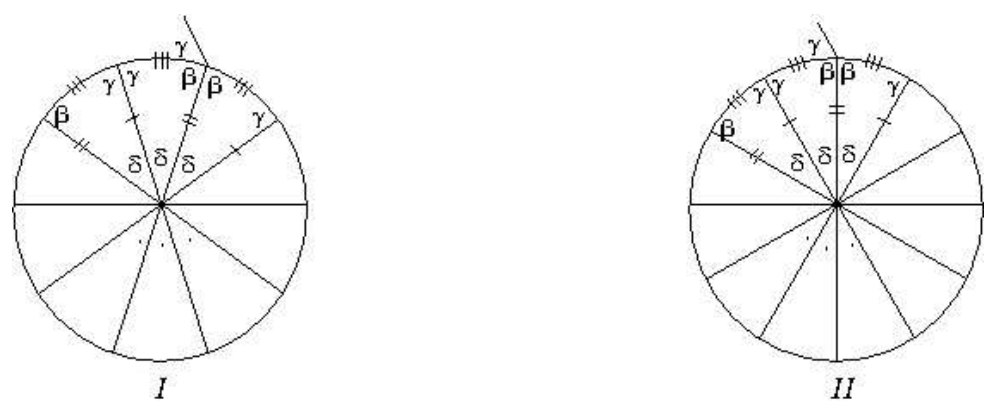

Figure 27: Angle arrangement at vertices with the sum $\alpha+q \delta+\gamma=\pi, q=3,4$.

1.2 Suppose now that $\gamma<\frac{\pi}{3}$, with $\theta_{3}=\gamma$ (Figure 19). Then, in order to fulfill the angle folding relation, the sum $3 \gamma$ must contain another parameter $\rho$ being a sum of angles, which does not contain $\beta$ and $\alpha$ (since the angular sequence $(\gamma, \gamma, \gamma, \gamma, \gamma, \beta, \alpha, \gamma)$ does not satisfy the angle folding relation). We shall study the cases $\rho=k \gamma, k=1,2, \rho=\gamma+\delta$ and $\rho=\delta, \rho=2 \delta$ separately.

1.2.1 Suppose $\rho=\gamma$. If $4 \gamma=\pi$, then $\delta>\frac{\pi}{12}$, since $\gamma+\delta>\frac{\pi}{3}$.

The sum of the alternate angles $\alpha$ and $\delta$ must satisfy $\alpha+t \delta=\pi, t=2, \ldots, 7$ or $2 \alpha+p \delta=\pi, p=1,2,3$ or $\alpha+\delta+2 \gamma=\pi$ or $\alpha+k \delta+\gamma=\pi, k=2,3,4$ (observe that if $k=1$, then $\delta>\frac{\beta}{4}=\gamma$ ). By the adjacency condition (1.1), the first case is valid for $t=3, \ldots, 7$, but expanding the angle arrangement, we always end up at a vertex surrounded by angles $\beta, \beta, \gamma$, whose sum $\beta+\gamma$ does not satisfy the angle folding relation, 
since $\gamma<\alpha$.

In the second case, we conclude that for $p=1, \delta \approx 46.62^{\circ}$, which is impossible since $\delta<\gamma$. Therefore, $p=2,3$ and once again the angle arrangement leads us to a vertex surrounded by angles $\beta, \beta, \gamma$ (whether $p=2$ or $p=3$ ) and so it is impossible to extend the configuration.

In the third case, the angles arrangement is $(\alpha, \delta, \delta, \beta, \gamma, \gamma, \gamma, \delta)$ and the sum $\delta+\beta+\gamma+\delta$ violates the angle folding relation. It remains the last case and if $\alpha+k \delta+\gamma=\pi, k=2,3,4$, respectively, we get $\alpha \approx 65.56^{\circ}, \delta \approx 34.72^{\circ}, \beta \approx 114.44^{\circ}$ or $\alpha \approx 63.27^{\circ}, \delta \approx 23.91^{\circ}, \beta \approx$ $116.73^{\circ}$ or $\alpha \approx 61.43^{\circ}, \delta \approx 18.39^{\circ}, \beta \approx 118.57^{\circ}$. The other sum of alternate angles at vertices surrounded by angles $\alpha, \delta$ and $\gamma$ is always (independently of the position of the $\left.k \delta^{\prime} s\right) \alpha+k \delta+\gamma=\pi$ or $\beta+(k+1) \delta=\pi$.

Taking into account the angles obtained by the adjacency condition, we conclude that the only possible sum is $\alpha+k \delta+\gamma=\pi, k=2,3,4$. Assuming that $k=2$, we may expand the configuration in Figure 19 and obtain a global representation of a f-tiling $\tau \in \Omega\left(T_{1}, T_{2}\right)$ (see Figure 28). Note that in the construction of the global representation, we must avoid the appearance of one angle $\beta$ at vertices that already have two angles $\beta$, since it leads to a configuration with a vertex in which one of its sum of alternate angles contains two angles $\beta$. This avoidance obliges tile 11 to be an equilateral triangle. The corresponding tiling has sixteen equilateral triangles and thirty-two scalene triangles and is denoted by $\mathcal{L}$.

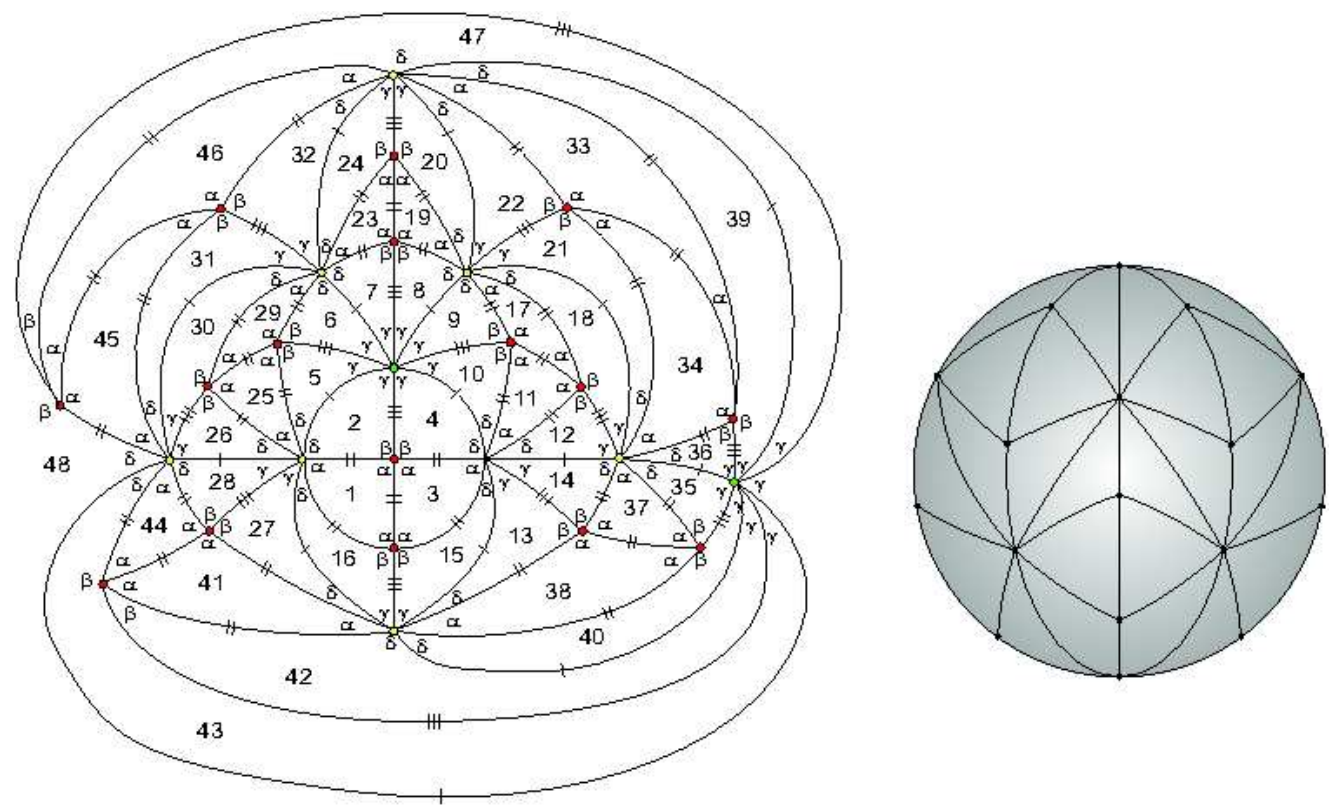

Figure 28: 2D and 3D representation of $\mathcal{L}$.

If $\alpha+k \delta+\gamma=\pi$, for $k=3$ and $k=4$, we always end up at a vertex surrounded by angles $\beta, \beta, \gamma$, since the angle arrangement at vertices of valency ten and twelve with this type of alternate sum has always three angles $\delta$ in consecutive positions, as in the case 1.1.2.3. 
1.2.2 If $5 \gamma=\pi$, then $\delta>\frac{2 \pi}{15}$ and again one of the sums at vertices surrounded by alternate angles $\alpha$ and $\delta$ must satisfy $\alpha+t \delta=\pi, \quad t=2,3,4$ or $2 \alpha+p \delta=\pi, p=1,2$ or $\alpha+\delta+2 \gamma=\pi$ or $\alpha+k \delta+\gamma=\pi, \quad k=1,2,3$.

1.2.2.1 In the first case, since $\gamma>\delta$, we get $t=4$ and so $\delta \approx 29.61^{\circ}, \alpha \approx 61.56^{\circ}, \beta \approx$ $118.44^{\circ}$. However, expanding the configuration in Figure 19, we end up at a vertex surrounded by a sequence of angles of the form $(\ldots, \beta, \beta, \gamma, \ldots)$ and so the sum $\beta+\gamma+\mu$ violates the angle folding relation, where $\mu$ is a sum of angles.

1.2.2.2 In the second case, for $p=1$, we get $\alpha \approx 64.29^{\circ}$ and $\delta \approx 51.43^{\circ}$ which is impossible (since $\delta<\gamma$ ); for $p=2$ we get $\alpha \approx 61.31^{\circ}, \delta \approx 28.69^{\circ}, \beta \approx 118.69^{\circ}$. The configuration extends a bit more and the next figure shows the possible positions of the angles arrangement surrounding vertices in which one of sums of alternate angles is $2 \alpha+2 \delta$. A contradiction is achieved in the configuration in Figure 29-I, II and III, since it always reaches at a vertex surrounded by angles $\beta, \beta, \gamma$ or a vertex surrounded by angles $\beta, \alpha, \beta$, whose sum $\beta+\gamma$ or $2 \beta$ does not satisfy the angle folding relation.

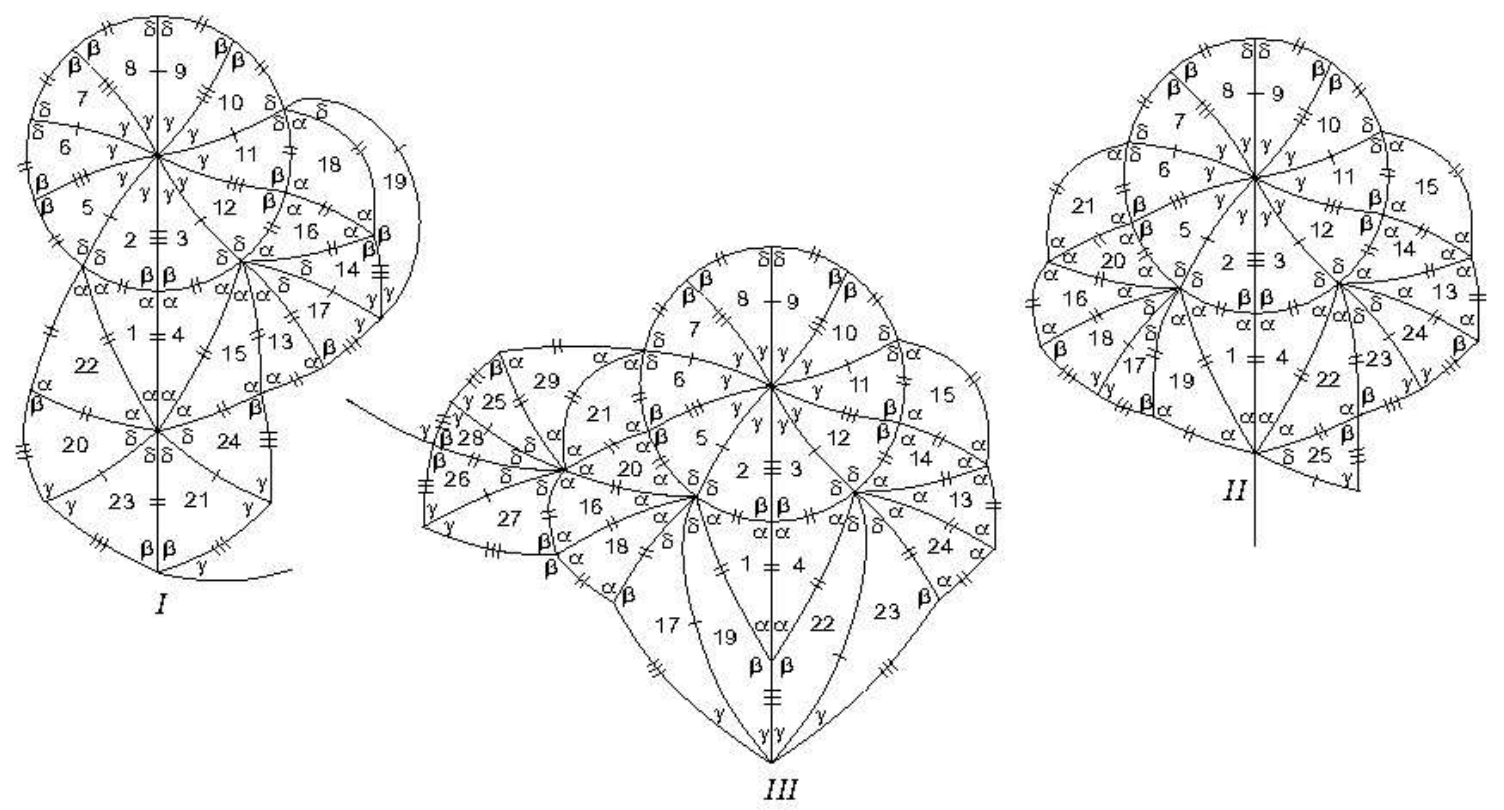

Figure 29: Local configuration.

1.2.2.3 If $\alpha+\delta+2 \gamma=\pi$, then, by the adjacency condition $(1.1), \delta \approx 44.1^{\circ}$, which is impossible.

1.2.2.4 If $\alpha+k \delta+\gamma=\pi$, for $k=1,2,3$, respectively, we get $\delta \approx 81.19^{\circ}$ or $\delta \approx 40.28^{\circ} \delta \approx$ $27.62^{\circ}$. Thus, for $k=1,2, \delta>\gamma$, which is a contradiction. Summarizing, $k=3$ and $\alpha \approx 61.15^{\circ}, \beta \approx 118.85^{\circ}, \delta \approx 27.62^{\circ}$. Extending the configuration in Figure 19 and choosing for tile 24 one of its two possible positions, we end up at a vertex surrounded by the angular sequence $(\beta, \beta, \gamma, \ldots)$, whose sum is $\beta+\gamma$ or $\beta+\gamma+\mu$, where $\mu$ is a sum of angles. In the first case, we conclude that $\gamma=\alpha$, which is impossible and in the second 
case, the sum violates the angle folding relation, Figure 30-I, II. The other position for tile 24 ends up in a similar impossibility.
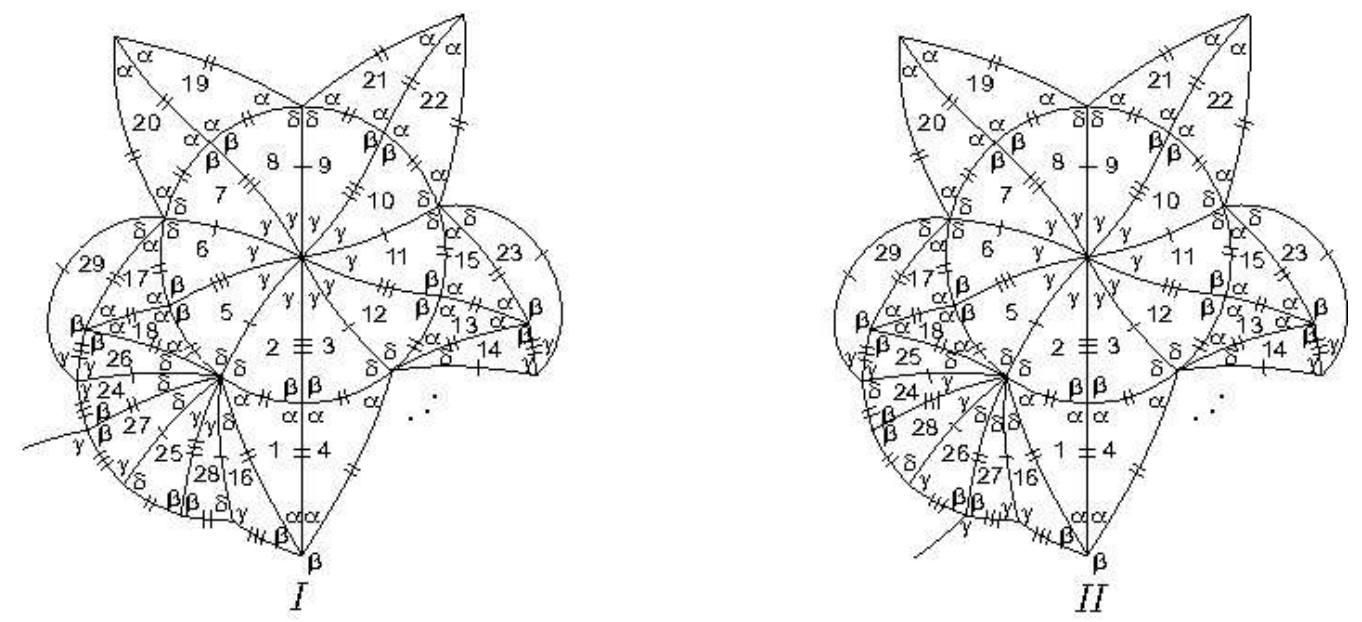

Figure 30: Local configuration.

1.2.3 The vertices of valency ten in which one of the sums of alternate angles is $4 \gamma+\delta=\pi$ gives rise to another vertex surrounded by one alternate angle $\beta$ and one angle $\gamma$, whose sum does not satisfy the angle folding relation, see Figure 31-I and II.
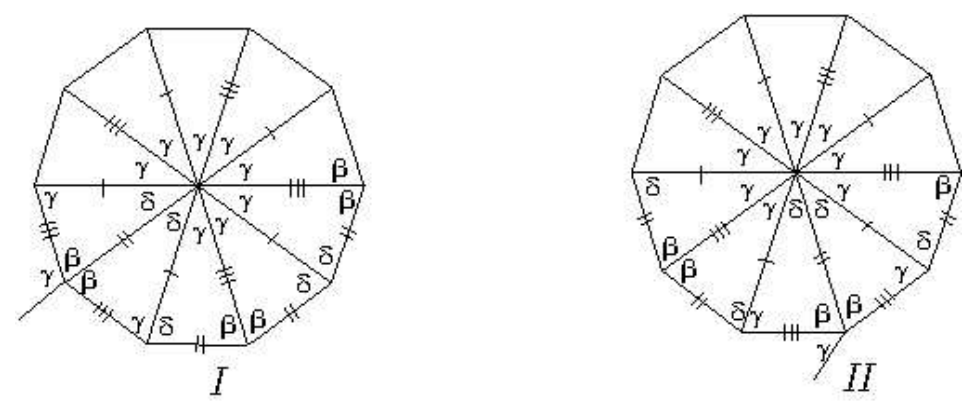

Figure 31: Angle arrangement around vertices satisfying $4 \gamma+\delta=\pi$.

1.2.4 Suppose $\rho=\delta$. Then, $3 \gamma+\delta=\pi$ and the configuration in Figure 19 ends up in the one shown in Figure 32-I. Once again we get an impossibility at vertex $v_{3}$.

1.2.5 If $\rho=2 \delta$, then $3 \gamma+2 \delta=\pi$. The configuration ends up in a vertex surrounded by angles $\beta$ and $\gamma$, similar to the one in Figure 31 and a contradiction is achieved as is shown in Figure 32-II. 

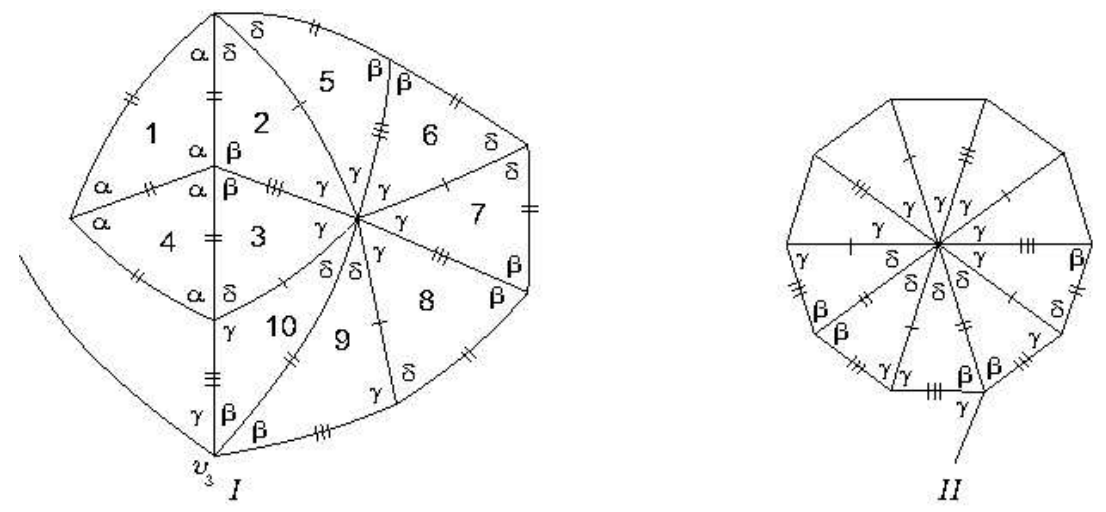

Figure 32: Local configuration.

1.3 Suppose now that $\theta_{3}=\delta$ (see Figure 19). Then, $2 \gamma+\delta \leq \pi$ and if $2 \gamma+\delta=\pi$, the configuration is given in Figure 33.

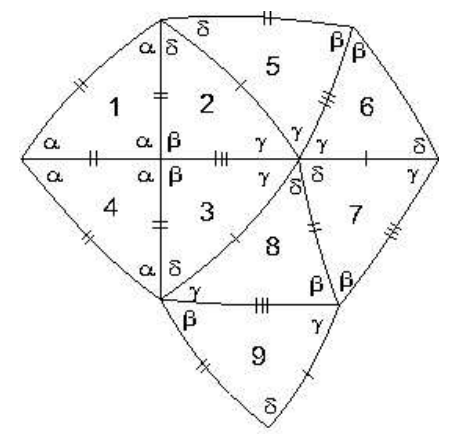

Figure 33: Local configuration.

The vertices surrounded by angles $\beta$ and $\gamma$ must be of valency four, so $\gamma=\alpha$ and consequently by the adjacency condition (1.1), $\alpha=\frac{\pi}{2}$, which is impossible. Therefore, $2 \gamma+\delta<\pi$ and a decision must be taken about the angle $\theta_{5} \in\{\delta, \alpha\}$ (see Figure 34).

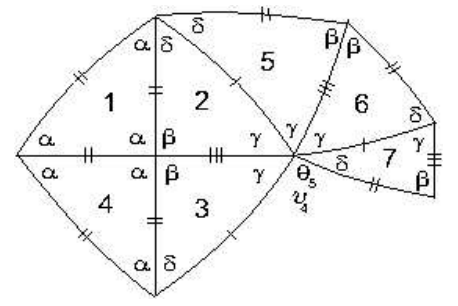

Figure 34: Local configuration. 
In case $\theta_{5}=\delta$, the configuration extends to the one shown in Figure 35.

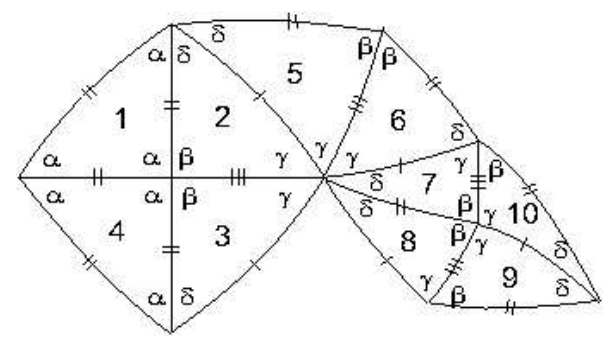

Figure 35: Local configuration.

The vertex surrounded by angles $\beta$ and $\gamma$ must be of valency four and once again $\gamma=\alpha$, which is impossible by the adjacency condition (1.1). Accordingly, $\theta_{5}=\alpha$ and since $2 \gamma+\alpha<\pi$ (due to edge compatibility), one has $3 \gamma+\alpha=\pi$ or $2 \gamma+\alpha+\delta=\pi$, taking into account that $\gamma>\frac{\pi}{6}$ and $\gamma+\delta>\alpha$.

If $3 \gamma+\alpha=\pi$, then $\delta>\frac{\pi}{9}$ and the other sum of alternate angles at vertex $v_{4}$ is of the form $\beta+\delta+2 \gamma=\pi$, which is impossible.

If $2 \gamma+\alpha+\delta=\pi$, we may add some new cells to the local configuration illustrated in Figure 35 and obtain the one in Figure 36.

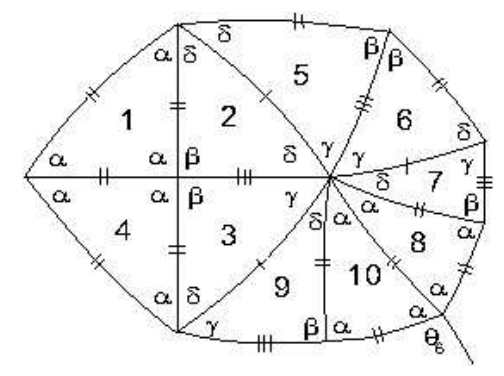

Figure 36: Local configuration.

The angle $\theta_{6}$ must be $\alpha$ or $\beta$. In case $\theta_{6}=\alpha$, then the sum containing two alternate angles $\alpha$ is $2 \alpha+\gamma=\pi$ or $2 \alpha+p \delta=\pi, p \geq 1$. However, by the assumption $2 \gamma+\alpha+\delta=\pi$, it is impossible that $2 \alpha+\gamma=\pi$ (note that $\gamma+\delta>\alpha$ ). Therefore, $2 \alpha+p \delta=\pi$ for some $p \geq 1$. The configuration extends and we obtain the one in Figure 37-I.

The vertices surrounded by alternate angles $\beta$ and $\gamma$, once again are of valency four, which is impossible since $\gamma=\alpha$ does not satisfy the condition $\alpha+\delta+2 \gamma=\pi$.

If $\theta_{6}=\beta$, then the configuration extends a bit more, but we end up again at a vertex surrounded by alternate angles $\beta$ and $\gamma$, which must be of valency four and consequently $\gamma=\alpha>\frac{\pi}{3}$, contradicting the assumption $2 \gamma+\alpha+\delta=\pi$ (Figure 37-II). 

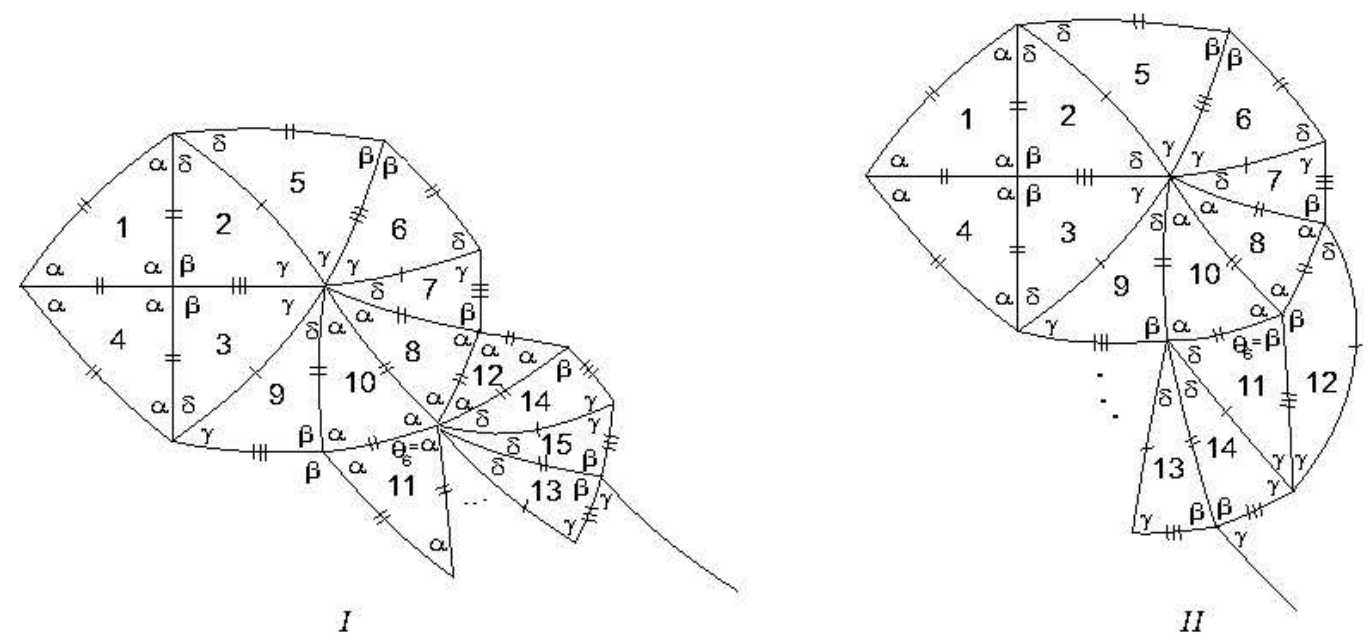

Figure 37: Local configuration.

2. Suppose now that $\theta_{1}=\delta$ (see Figure 17). If $\gamma+\delta=\pi$, then $\beta>\gamma>\frac{\pi}{2}$ and from the assumption $\alpha+\beta=\pi$, then $\delta, \alpha<\frac{\pi}{2}$. However, the configuration can not be expanded since the sum $\rho+\beta$ (see Figure 38) does not satisfy the angle folding relation, for any $\rho \in\{\gamma, \beta\}$.

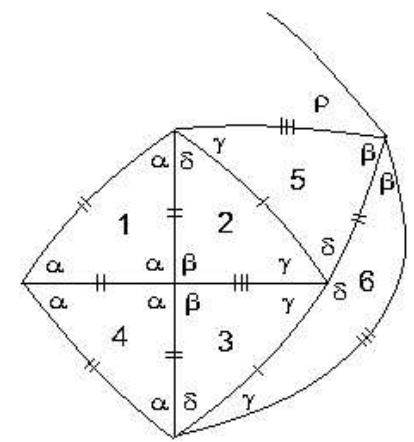

Figure 38: Local configuration.

As $\gamma+\delta<\pi$, then $\delta<\frac{\pi}{2}$. If $\gamma \geq \frac{\pi}{2}$, then $\beta>\frac{\pi}{2}$ and $\alpha<\frac{\pi}{2}$, which is impossible by the adjacency condition (1.1). Therefore, $\gamma<\frac{\pi}{2}$ and also $\alpha<\frac{\pi}{2}<\beta$, by the adjacency condition (1.1).

The configuration started in Figure 17 extends to the one in Figure 39. 


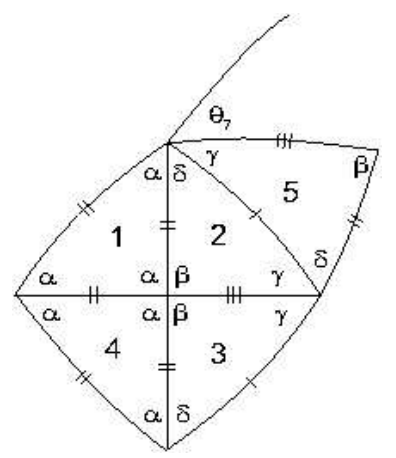

Figure 39: Local configuration.

The angle labelled $\theta_{7}$ is either $\beta$ or $\gamma$.

2.1 Suppose firstly, that $\theta_{7}$ is $\beta$. Therefore, in order to satisfy the angle folding relation, the sum containing alternate angles $\beta$ and $\delta$ is $\beta+r \delta=\pi$, for some $r>1$. The other sum of alternate angles at the same vertex is $\alpha+\gamma+(r-1) \delta$, as is illustrated in the Figure 40 .

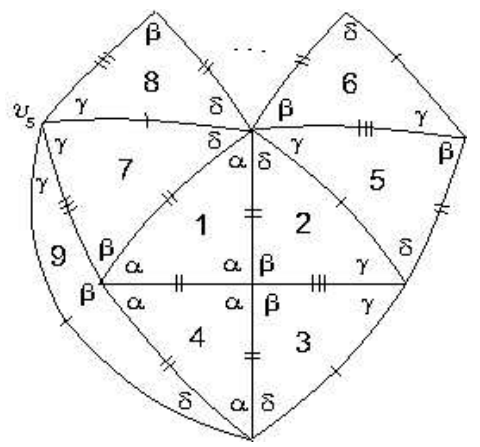

Figure 40: Local configuration.

Looking at vertex $v_{5}$ surrounded only by angles $\gamma$, one of the sums of alternate angles is $2 \gamma+\lambda$, where the parameter $\lambda$ is a sum of angles not containing any $\beta$, due to the angle folding relation.

2.1.1 Suppose that $\lambda=\alpha$. Then, $2 \gamma+\alpha \leq \pi$. However, the case $2 \gamma+\alpha=\pi$ is impossible, since the other sum of alternate angles is $\beta+\gamma+\delta$, not satisfying the angle folding relation, as is illustrated in the Figure 41.

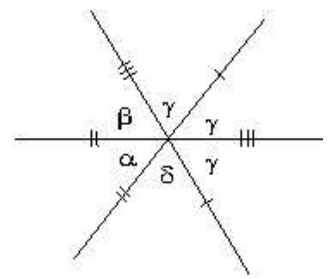

Figure 41: Angle arrangement. 
As $2 \gamma+\alpha<\pi$, then $3 \gamma+\alpha=\pi$ or $2 \gamma+\alpha+\delta=\pi$. If $3 \gamma+\alpha=\pi$, having in account that $\alpha+\gamma+(r-1) \delta=\pi$ and $\beta+r \delta=\pi$, we conclude that $\beta+\delta+2 \gamma=\pi$, which is a contradiction.

Also, if $2 \gamma+\delta+\alpha=\pi$, for the same reason $\beta+\gamma+2 \delta=\pi$, which is again an impossibility.

2.1.2 Suppose that $\lambda=m \gamma, m=1,2,3$. If $m=1$, then $3 \gamma=\pi$ and the angle arrangement around vertex $v_{5}$ is illustrated in the Figure 42 .

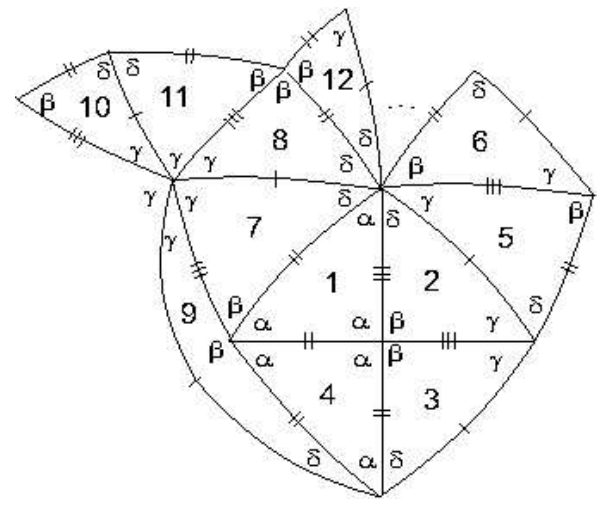

Figure 42: Local configuration.

Observing tiles labelled 8,11 and 12 and the position of the angle $\beta$, we can not expand the configuration, since $\beta>\frac{\pi}{2}$.

If $m=2,3$, then $4 \gamma=\pi$ and $5 \gamma=\pi$, but we are led to the same contradiction illustrated in Figure 42.

2.1.3 If $\lambda=\gamma+k \delta, k=1,2$, then $3 \gamma+k \delta=\pi$ and the configuration ends up at a vertex surrounded by angles $\gamma, \delta$ and $\beta$, which is impossible since the sum of the alternate angles $\beta$ and $\gamma$ does not satisfy the angle folding relation (see Figure 43).

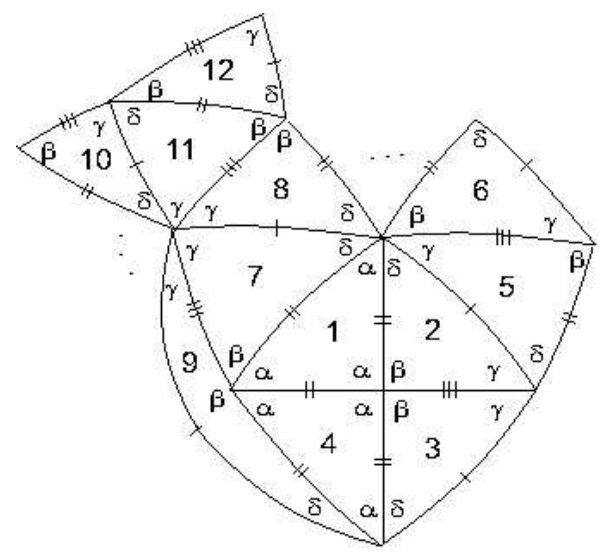

Figure 43: Local configuration. 
2.1.4 Suppose that $\lambda=\delta$. If the sum $2 \gamma+\delta$ satisfies the angle folding relation, then $\gamma>\frac{\pi}{3}$ and the local representation in Figure 39 extends to the one illustrated in Figure 44-I.
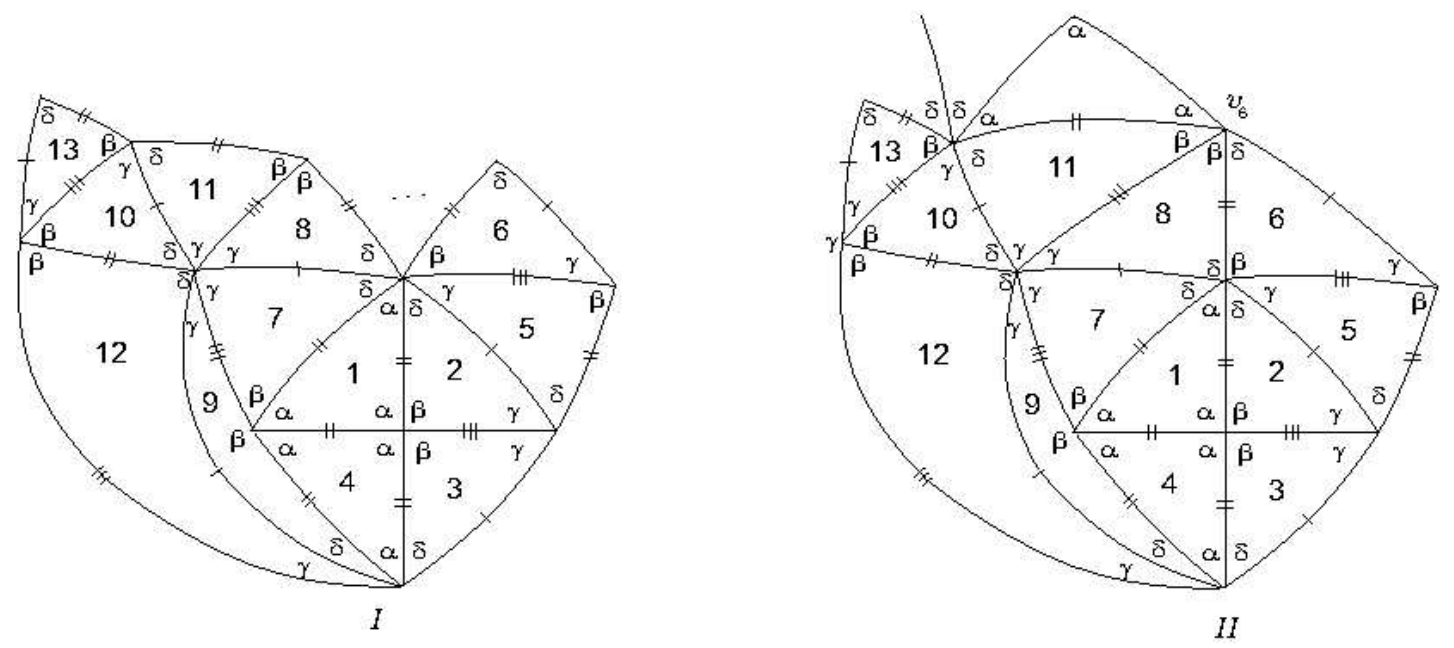

Figure 44: Local configuration.

The vertices surrounded by alternate angles $\beta$ and $\gamma$ must be of valency four, for which $\gamma=\alpha$ and from the assumption in 2.1, $r=2$, i.e. $\beta+2 \delta=\pi=\alpha+\gamma+\delta$. Figure 44-II illustrates the expanded configuration and looking at vertex $v_{6}$, we conclude that is of valency four, which is impossible since $\delta<\gamma=\alpha$. Therefore, $2 \gamma+\delta<\pi$ and so, $2 \gamma+k \delta=\pi$, for some $k \geq 2$. However, this case is similar to the case $2 \gamma+\delta=\pi$.

2.2 Suppose that $\theta_{7}=\gamma$ (see Figure 39). Consequently $\alpha+\gamma+\rho=\pi$, for some $\rho$ different from $\beta$.

2.2.1 If $\rho=\alpha$, then we have $2 \alpha+\gamma=\pi$, since $\gamma+\delta>\alpha>\frac{\pi}{3}$. However, due to the edge compatibility, it is impossible to arrange the angles in order to satisfy $2 \alpha+\gamma=\pi$.

2.2.2 If $\rho=\gamma$, then $\alpha+2 \gamma<\pi$, since $\alpha+2 \gamma=\pi$ implies that the other sum of alternate angles is $\beta+\gamma+\delta=\pi$, which is an impossibility. Taking into account that, $\gamma+\delta>\alpha>\frac{\pi}{3}$ and $\gamma>\frac{\pi}{6}$, one has $\alpha+3 \gamma=\pi$ or $\alpha+2 \gamma+\delta=\pi$. Again, by the angle arrangement, the case $\alpha+3 \gamma=\pi$ leads us to the sum $\beta+\delta+2 \gamma=\pi$, which is impossible. Therefore, $\alpha+2 \gamma+\delta=\pi$ and we may add some new cells to the configuration in Figure 39. Choosing for tile 7 one of its two possible positions, we end up at the configuration in Figure 45.

Looking at vertex labelled $v_{7}$, we conclude that it must be of valency four and therefore $\gamma=\alpha>\frac{\pi}{3}$, contradicting the sum $\alpha+2 \gamma+\delta=\pi$. 


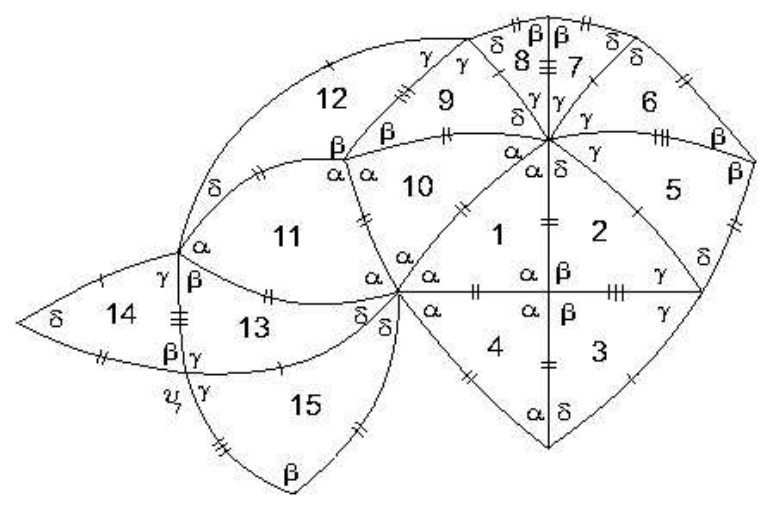

Figure 45: Local configuration.

The other position for tile numbered 7 leads us to a contradiction, Figure 46, since $\xi=\beta$ or $\zeta=\beta$.

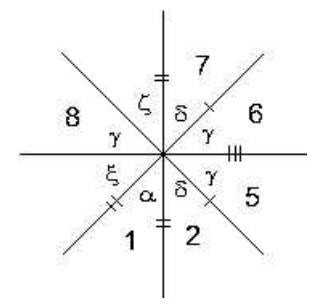

Figure 46: Angle arrangement.

2.2.3 If $\rho=\delta$, then $\alpha+\gamma+\delta \leq \pi$. We shall study the cases $\alpha+\gamma+\delta=\pi$ and $\alpha+\gamma+\delta<\pi$ separately.

2.2.3.1 Suppose, firstly, that $\alpha+\gamma+\delta=\pi$. Taking into account that $\gamma+\delta>\alpha$, one has $\gamma>\frac{\pi}{4}$. The local configuration in Figure 39 extends a bit more to the one in Figure 47.

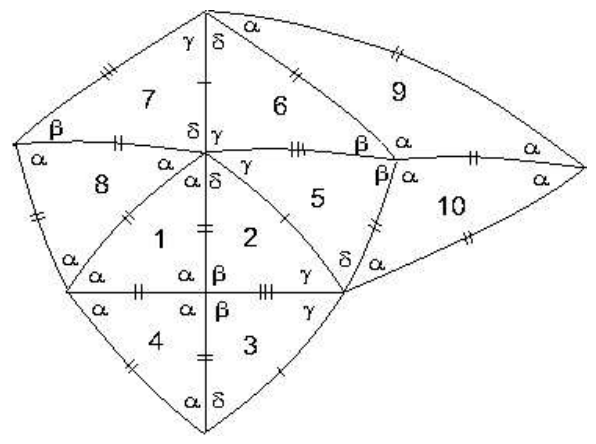

Figure 47: Local configuration. 
In order to satisfy the angle folding relation, the sum of the alternate angles $2 \alpha$ is $2 \alpha+\gamma=\pi$ or $2 \alpha+p \delta=\pi$, for some $p \geq 1$. If $2 \alpha+\gamma=\pi$, then $\delta=\alpha>\frac{\pi}{3}$ and consequently $\gamma>\frac{\pi}{3}$ not satisfying $\alpha+\gamma+\delta=\pi$. Therefore, $2 \alpha+p \delta=\pi, p \geq 1$ and, by the assumption $\alpha+\delta+\gamma=\pi$, we get $\alpha+(p-1) \delta=\gamma$. Consequently, $\gamma \geq \alpha>\frac{\pi}{3}$, which implies that the sum of alternate angles at vertices surrounded by $\alpha$ and $\gamma$ must satisfy $\alpha+\gamma+\delta=\pi$. Accordingly, $\alpha+\beta=\pi, 2 \alpha+p \delta=\pi, p \geq 1$ and $\alpha+\gamma+\delta=\pi$. Expanding the configuration and attending to the choice of the edge sides of tile 13, we get the one shown in Figure 48. Looking at vertex $v_{8}$, the configuration cannot be extended, since the sum $2 \beta$ violates the angle folding relation.

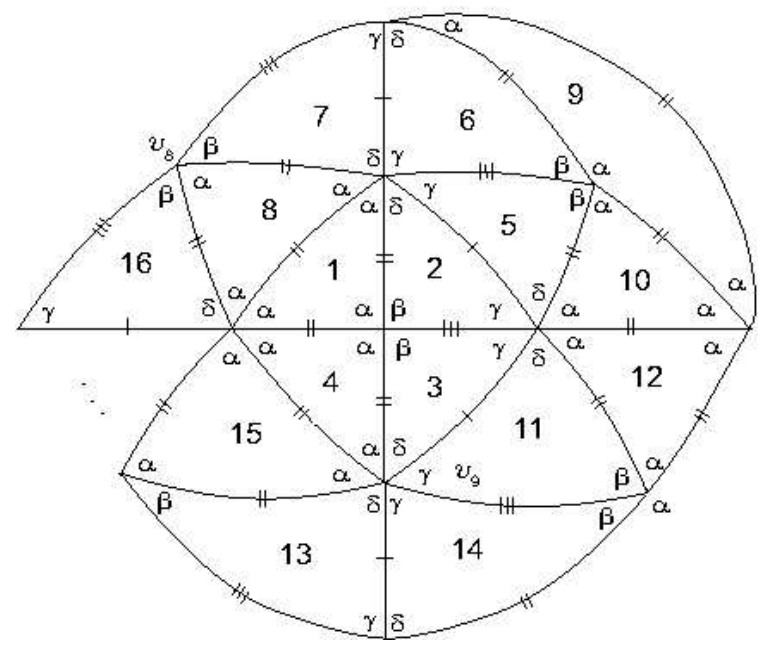

Figure 48: Local configuration.

The other position of tile numbered 13 implies that, at vertex $v_{9}$, the sequence of angles is $(\alpha, \delta, \gamma, \beta, \delta, \delta)$ (Figure 49$)$.

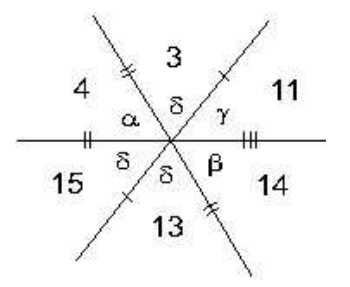

Figure 49: Angle arrangement.

Summarizing, $\alpha+\beta=\pi, \alpha+\gamma+\delta=\pi, \beta+2 \delta=\pi$ and $2 \alpha+p \delta=\pi, p \geq 1$, which implies that $\delta>\frac{\pi}{6}$ and $p=1$. Therefore, $\alpha=\gamma=\frac{2 \pi}{5}, \delta=\frac{\pi}{5}$ and $\beta=\frac{3 \pi}{5}$. The configuration extends to the following one and we are led to vertices surrounded by three angles $\beta$, whose sum $2 \beta$ violates the angles folding relation. 


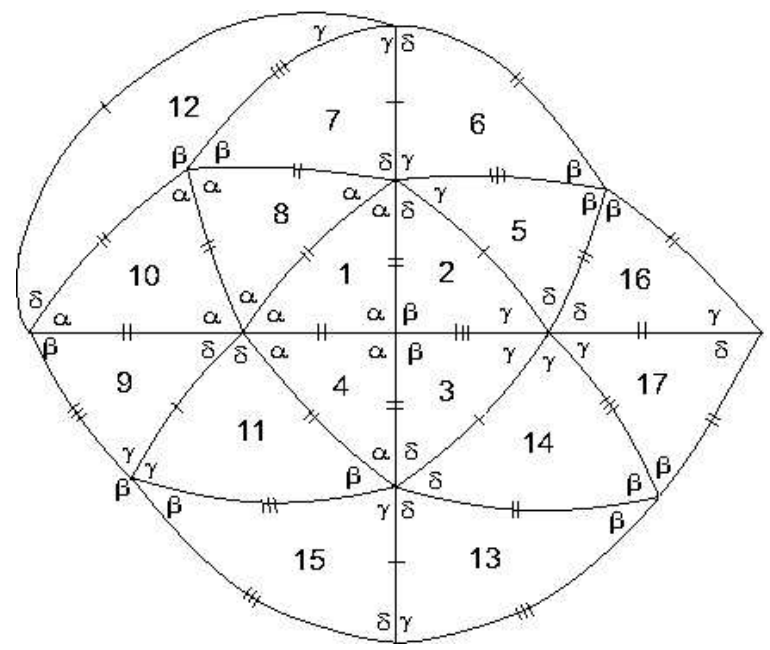

Figure 50: Local configuration.

2.2.3.2 Suppose now that $\alpha+\gamma+\delta<\pi$. Then, $\alpha+2 \gamma+\delta=\pi$ or $\alpha+\gamma+r \delta=\pi$, for some $r \geq 1$. The first case is similar to the one studied in $\mathbf{2 . 2 . 2}$.

If $\alpha+\gamma+r \delta=\pi, r \geq 1$, the configuration in Figure 39 can be extended and we get the one in Figure 51.

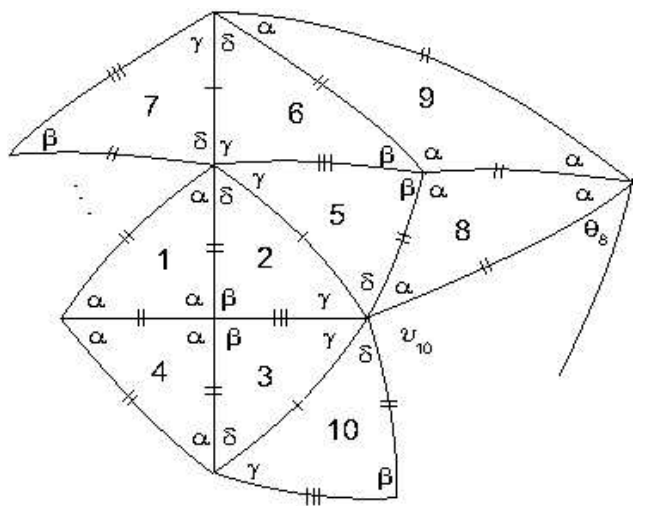

Figure 51: Local configuration.

A decision about the angle labelled $\theta_{8} \in\{\alpha, \beta\}$ must be taken.

2.2.3.2.1 Assuming that $\theta_{8}=\alpha$, the sum containing two alternate angles $\alpha$ must satisfy $2 \alpha+p \delta=\pi$, for some $p \geq 1$, otherwise it would satisfy $2 \alpha+\gamma=\pi$, and consequently, by the adjacency rules for the sides, the other sum would be $\beta+\alpha+\delta=\pi$, which is impossible. Therefore, the configuration extends a bit more to the one illustrated in Figure 52. Note that to avoid the appearance of one angle $\beta$ at vertex $v_{11}$, the sides of tile 12 must be in the position illustrated. Looking at the angle $\omega=\beta$, we conclude that the configuration below can not be extended. 


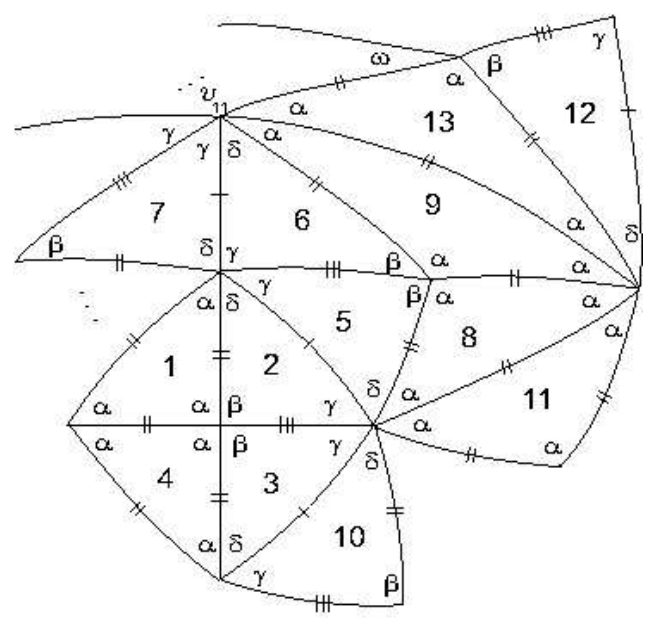

Figure 52: Local configuration.

2.2.3.2.2 If $\theta_{8}=\beta$, the vertex labelled $v_{10}$ is as illustrated in Figure 53 and we conclude that the other sum of alternate angles satisfies $\gamma+(r+1) \delta=\pi$.

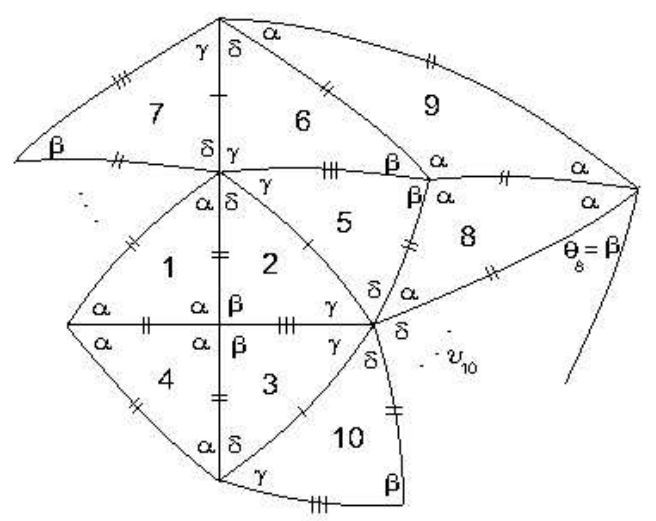

Figure 53: Local configuration.

Consequently $\delta=\alpha<\gamma$, which is impossible.

Proposition 2.4. If $x=\beta$ and $\alpha+x<\pi$, then $\Omega\left(T_{1}, T_{2}\right)=\varnothing$.

Proof. Suppose that $x=\beta$ (see Figure 2) and $\alpha+x<\pi$. Then, $\gamma+\delta>\alpha>\frac{\pi}{3}, \gamma>\frac{\pi}{6}$ and consequently $\alpha+\beta+\gamma=\pi$ or $\alpha+\beta+t \delta=\pi$, for some $t \geq 1$.

1. Suppose that $\alpha+\beta+\gamma=\pi$. Then, the configuration in Figure 2 extends to the one illustrated in Figure 54 and tile 4 has two possible positions. Either way, the other sum of alternate angles satisfies $2 \beta+\delta=\pi$, which is impossible since $\beta+\gamma+\delta>\pi$.

2. If $\alpha+\beta+t \delta=\pi$, for some $t \geq 1$ and since $\beta+\gamma+\delta>\pi$, one has $\gamma>\alpha>\frac{\pi}{3}$. 

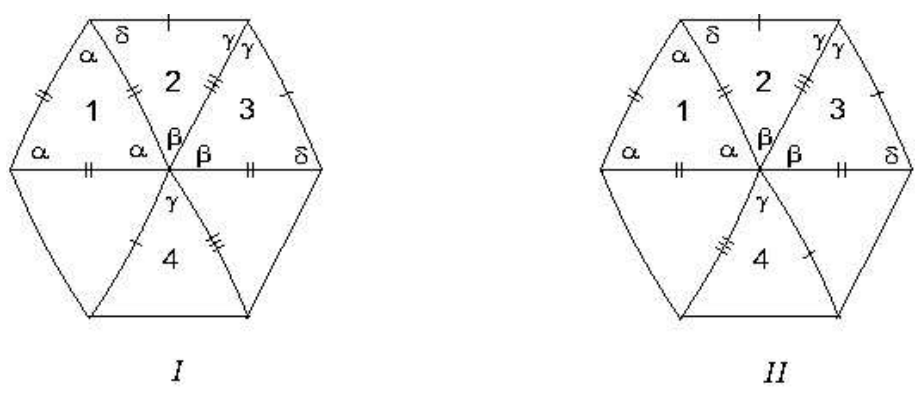

Figure 54: Local configuration.

Assuming that $\gamma \geq \frac{\pi}{2}$, then, $\beta>\frac{\pi}{2}$ and $\alpha<\frac{\pi}{2}$, which contradicts the adjacency condition (1.1). Therefore, $\delta<\gamma<\frac{\pi}{2}, \alpha<\frac{\pi}{2}$ and consequently $\beta \geq \frac{\pi}{2}$.

2.1 Assume firstly, that $\beta=\frac{\pi}{2}$. The configuration in Figure 2 extends to the one below and a decision must be taken about the angle $\theta_{1} \in\{\gamma, \delta\}$.

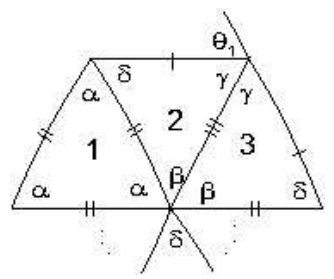

Figure 55: Local configuration.

2.1.1 If $\theta_{1}=\gamma$, then the sum containing two angles $\gamma$ is $2 \gamma+\delta=\pi$, since $\gamma>\frac{\pi}{3}$ and $\gamma+\delta>\frac{\pi}{2}$. Extending the configuration above, tile 8 has two possible positions as is shown in Figure 56-I and II.
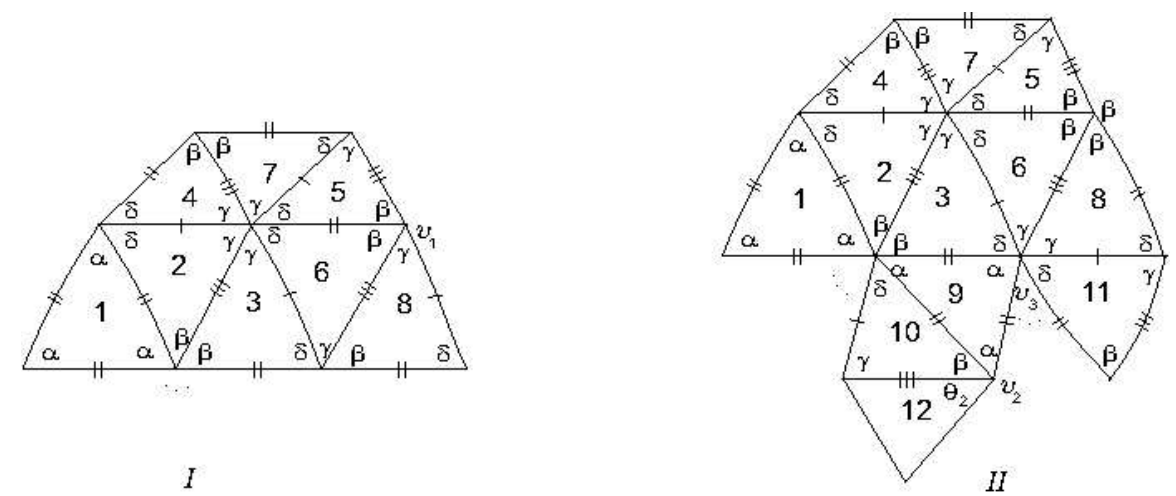

Figure 56: Local configuration. 
In Figure 56-I, the sum of the alternate angles $\beta$ and $\gamma$ at vertex $v_{1}$ must satisfy $\beta+\gamma=\pi$, which is impossible since $\beta=\frac{\pi}{2}$ and $\gamma<\beta$.

In Figure 56-II, the angle $\theta_{2}$ in tile 12 must be $\gamma$ or $\beta$. If $\theta_{2}=\gamma$, one of the sums of alternate angles at vertex $v_{2}$ satisfies $\alpha+\gamma+p \delta=\pi$ and the other $\beta+\delta+p \delta=\pi$, for some $p \geq 1$ (see Figure 57). From $\alpha+\gamma+p \delta=\beta+\delta+p \delta$, we conclude that $\alpha+\gamma=\beta+\delta$ and since $\gamma>\alpha$, then $\delta>\frac{\pi}{6}$, which contradicts the assumption $\alpha+\beta+t \delta=\pi, t \geq 1$. Therefore, $\theta_{2}=\beta$.

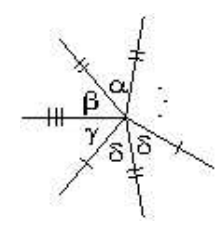

Figure 57: Angle arrangement at vertex $v_{2}$.

From the conditions, $\alpha+\beta+t \delta=\pi$ and $\alpha+\gamma+p \delta=\pi$, at vertex $v_{3}$, we get $\gamma+p \delta=\beta+t \delta$ and since $\gamma+\delta>\frac{\pi}{2}$, then $p<t+1$. On the other hand, from the same assumptions and since $\gamma<\beta$, we get $t<p$. Therefore, $t<p<t+1$, which is impossible.

2.1.2 If $\theta_{1}=\delta$, the configuration in Figure 55 is now,

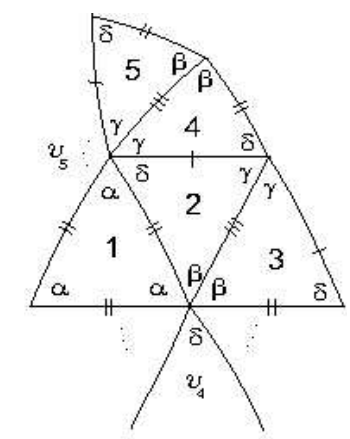

Figure 58: Local configuration.

Once again, by the conditions $\alpha+\beta+t \delta=\pi$, at vertex $v_{4}$ and $\alpha+\gamma+p \delta=\pi$, at vertex $v_{5}$, a contradiction is achieved.

2.2 Assuming that $\beta>\frac{\pi}{2}$ and since $\alpha+\delta<\alpha+\gamma<\alpha+\beta<\pi, 2 \beta>\pi$ and $\delta<\gamma<\frac{\pi}{2}$, then vertices of valency four must be surrounded by alternate angles $\gamma$ and $\beta$ or $\delta$ and $\beta$.

2.2.1 Extending the same configuration in Figure 55 and if the angle labelled $\theta_{1}$ is $\gamma$, then one has $2 \gamma+q \delta=\pi$, for some $q \geq 1$ and we get the one illustrated in Figure 59 . 


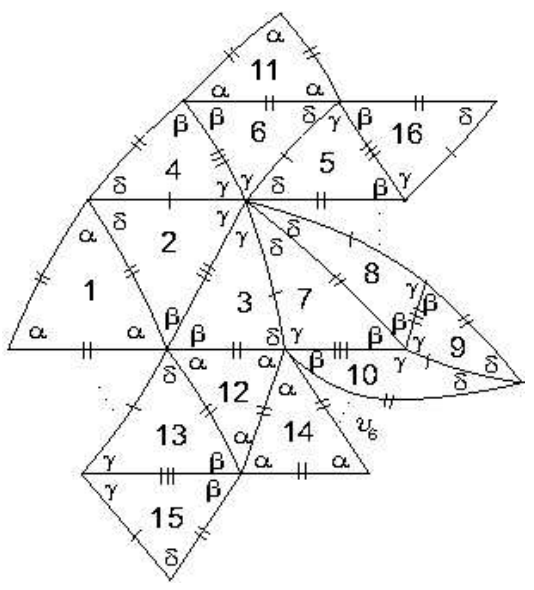

Figure 59: Local configuration.

Both sums of the alternate angles at vertex $v_{6}$ are $\alpha+\gamma+p \delta=\pi$ and $\alpha+\beta+p \delta=\pi$, which is a contradiction, since $\gamma<\beta$.

2.2.2 Suppose finally that $\theta_{1}=\delta$ (see Figure 55). The sum containing $\alpha$ and $\gamma$ must satisfy $\alpha+\gamma+p \delta=\pi$, for some $p \geq 1$ and the configuration is the one illustrated in Figure 60 .

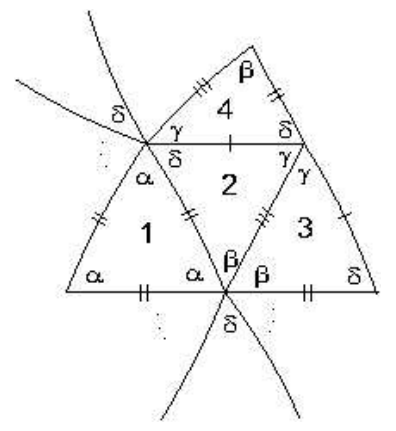

Figure 60: Local configuration.

From the sums $\alpha+\gamma+p \delta=\pi$ and $\alpha+\beta+t \delta=\pi$ assumed in 2., we get the same contradiction as in 2.1.1.

\section{$3 \quad$ Symmetry Groups}

Here we present the group of symmetries of the spherical f-tilings obtained $\mathcal{E}_{\alpha}, \mathcal{G}^{k}, k \geq$ $1, \mathcal{E}, \mathcal{F}, \mathcal{H}$ and $\mathcal{L}$. We also indicate the transitivity classes of isogonality and isohedrality.

In Table 1 is shown a complete list of all spherical dihedral f-tilings, whose prototiles are an equilateral triangle $T_{1}$ of angle $\alpha$ and a scalene triangle $T_{2}$ of angles $\delta, \gamma, \beta$, $(\delta<\gamma<\beta)$. 
We have used the following notation:

- $M$ and $N$ are, respectively, the number of triangles congruent to $T_{1}$ and the number of triangles congruent to $T_{2}$ used in such dihedral f-tilings;

- $G(\tau)$ is the symmetry group of the f-tiling $\tau$. The numbers of isohedrality-classes and isogonality-classes for the symmetry group are denoted, respectively, by \# isoh. and \# isog.;

- By $C_{n}$ and $D_{n}$ we denote, respectively, the cyclic group of order $n$ and the dihedral group of order $2 n$;

- $\beta=\beta_{\alpha}$, in f-tiling $\mathcal{E}_{\alpha}$, is given by

$$
\frac{\cos \alpha(1+\cos \alpha)}{\sin ^{2} \alpha}=\frac{\cos \gamma+\cos \delta \cos \beta}{\sin \delta \sin \beta},
$$

with $\gamma=\pi-\alpha$ and $\delta=\pi-\beta$;

- $\delta=\delta_{1}^{k}$, in f-tiling $\mathcal{G}^{k}$, is the solution of

$$
\frac{\cos \alpha(1+\cos \alpha)}{\sin ^{2} \alpha}=\frac{\cos \gamma+\cos \delta \cos \beta}{\sin \delta \sin \beta},
$$

with $\alpha=\pi-(2 k+1) \delta, \gamma=(k+1) \delta$ and $\beta=\pi-(k+1) \delta$.

\begin{tabular}{|c|c|c|c|c|c|c|c|}
\hline f-tiling & $\alpha$ & $\delta$ & $\gamma$ & $\beta$ & $G(\tau)$ & $\#$ isoh. & \# isog. \\
\hline \hline $\mathcal{E}_{\alpha}$ & ]$\frac{\pi}{2}, \pi[$ & $\pi-\beta$ & $\pi-\alpha$ & $\beta_{\alpha}$ & $D_{3}$ & 2 & 1 \\
\hline $\mathcal{G}^{k}, k \geq 1$ & $\pi-(2 k+1) \delta$ & $\delta_{1}^{k}$ & $(k+1) \delta$ & $\pi-(k+1) \delta$ & $D_{3}$ & $2 k+2$ & $k+1$ \\
\hline $\mathcal{E}$ & $72.75^{\circ}$ & $53.6^{\circ}$ & $\frac{\pi}{3}$ & $107.25^{\circ}$ & $C_{2} \times D_{3}$ & 2 & 3 \\
\hline $\mathcal{F}$ & $\frac{2 \pi}{5}$ & $\frac{\pi}{5}$ & $\frac{\pi}{3}$ & $\frac{3 \pi}{5}$ & $D_{6}$ & 3 & 3 \\
\hline $\mathcal{H}$ & $70.52^{\circ}$ & $24.74^{\circ}$ & $\frac{\pi}{3}$ & $109.48^{\circ}$ & $D_{6}$ & 4 & 3 \\
\hline $\mathcal{L}$ & $65.56^{\circ}$ & $34.72^{\circ}$ & $\frac{\pi}{4}$ & $114.44^{\circ}$ & $D_{8}$ & 4 & 3 \\
\hline
\end{tabular}

Table 1: The Combinatorial Structure of the Dihedral f-Tilings of the Sphere by Equilateral and Scalene Triangles with adjacency of type III

In Table 2 is shown a complete list of all spherical dihedral f-tilings, whose prototiles are an equilateral triangle $T_{1}$ of angle $\alpha$ and a scalene triangle $T_{2}$ of angles $\delta, \gamma, \beta$, $(\delta<\gamma<\beta)$ 
We have used the following notation.

- The angles $\delta$ and $\gamma$, in f-tiling $\mathcal{F}_{1}^{\delta}$, obey

$$
\frac{-\cos (\gamma+\delta)}{1+\cos (\gamma+\delta)}=\frac{\cos \gamma \cos \delta}{\sin \gamma \sin \delta}
$$

Besides, $\alpha=\pi-(\gamma+\delta)$;

- $\delta_{0} \approx 54.74^{\circ}$ and $\gamma_{0} \approx 70.53^{\circ}$;

- $\alpha=\alpha_{1}^{p}, \quad p \geq 4$, in f-tiling $\mathcal{D}^{p}$, is the solution of

$$
\frac{\cos \alpha(1+\cos \alpha)}{\sin ^{2} \alpha}=\frac{\cos \delta+\cos \gamma \cos \beta}{\sin \gamma \sin \beta},
$$

with $p \delta=\pi, \beta=\pi-\alpha$ and $\gamma=\pi-2 \alpha$;

- $\alpha=\alpha_{2}^{p}, \quad p \geq 4$, in f-tiling $\mathcal{F}^{p}$, is the solution of

$$
\frac{\cos \alpha(1+\cos \alpha)}{\sin ^{2} \alpha}=\frac{\cos \delta+\cos \gamma \cos \beta}{\sin \gamma \sin \beta},
$$

with $p \delta=\pi, \beta=\pi-\alpha$ and $\gamma=\frac{\pi}{2}-\frac{\alpha}{2}$;

- $\alpha=\alpha_{3}^{m}, m \geq 5$, in f-tiling $\mathcal{E}^{m}$, is the solution of

$$
\frac{\cos \alpha(1+\cos \alpha)}{\sin ^{2} \alpha}=\frac{\cos \delta+\cos \gamma \cos \beta}{\sin \gamma \sin \beta},
$$

with $m \delta=\pi, \beta=\pi-\alpha$ and $\gamma=\frac{\pi}{2}-\frac{\alpha}{2}-\frac{\pi}{2 m}$;

- $\beta=\beta_{\alpha}$, in f-tiling $\mathcal{E}_{\alpha}$, is given by

$$
\frac{\cos \alpha(1+\cos \alpha)}{\sin ^{2} \alpha}=\frac{\cos \gamma+\cos \delta \cos \beta}{\sin \delta \sin \beta},
$$

with $\gamma=\pi-\alpha$ and $\delta=\pi-\beta$;

- $\delta=\delta_{1}^{k}$, in f-tiling $\mathcal{G}^{k}$, is the solution of

$$
\frac{\cos \alpha(1+\cos \alpha)}{\sin ^{2} \alpha}=\frac{\cos \gamma+\cos \delta \cos \beta}{\sin \delta \sin \beta}
$$

with $\alpha=\pi-(2 k+1) \delta, \gamma=(k+1) \delta$ and $\beta=\pi-(k+1) \delta$. 


\begin{tabular}{|c|c|c|c|c|c|c|}
\hline f-tiling & $\alpha$ & $\delta$ & $\gamma$ & $\beta$ & $M$ & $N$ \\
\hline \hline $\mathcal{F}_{1}^{\delta}$ & ]$\gamma_{0}, \frac{\pi}{2}[$ & ] $0, \delta_{0}[$ & ]$\delta_{0}, \frac{\pi}{2}[$ & $\frac{\pi}{2}$ & 8 & 24 \\
\hline $\mathcal{F}_{2}^{\delta}$ & ]$\gamma_{0}, \frac{\pi}{2}[$ & ] $0, \delta_{0}[$ & ]$\delta_{0}, \frac{\pi}{2}[$ & $\frac{\pi}{2}$ & 8 & 24 \\
\hline \hline $\mathcal{D}^{p}, p \geq 4$ & $\alpha_{1}^{p}$ & $\frac{\pi}{p}$ & $\pi-2 \alpha$ & $\pi-\alpha$ & $4 p$ & $4 p$ \\
\hline $\mathcal{F}^{p}, p \geq 4$ & $\alpha_{2}^{p}$ & $\frac{\pi}{p}$ & $\frac{\pi}{2}-\frac{\alpha}{2}$ & $\pi-\alpha$ & $2 p$ & $4 p$ \\
\hline $\mathcal{E}^{m}, m \geq 5$ & $\alpha_{3}^{m}$ & $\frac{\pi}{m}$ & $\frac{\pi}{2}-\frac{\alpha}{2}-\frac{\pi}{2 m}$ & $\pi-\alpha$ & $4 m$ & $8 m$ \\
\hline \hline $\mathcal{E}_{\alpha}$ & ]$\frac{\pi}{2}, \pi[$ & $\pi-\beta$ & $\pi-\alpha$ & $\beta_{\alpha}$ & 2 & 6 \\
\hline $\mathcal{G}^{k}, k \geq 1$ & $\pi-(2 k+1) \delta$ & $\delta_{1}^{k}$ & $(k+1) \delta$ & $\pi-(k+1) \delta$ & 2 & $6(2 k+1)$ \\
\hline $\mathcal{E}$ & $72.75^{\circ}$ & $53.63^{\circ}$ & $\frac{\pi}{3}$ & $107.25^{\circ}$ & 6 & 12 \\
\hline $\mathcal{F}$ & $\frac{2 \pi}{5}$ & $\frac{\pi}{5}$ & $\frac{\pi}{3}$ & $\frac{3 \pi}{5}$ & 12 & 12 \\
\hline $\mathcal{H}$ & $70.52^{\circ}$ & $24.74^{\circ}$ & $\frac{\pi}{3}$ & $109.48^{\circ}$ & 12 & 24 \\
\hline $\mathcal{L}$ & $65.56^{\circ}$ & $34.72^{\circ}$ & $\frac{\pi}{4}$ & $114.44^{\circ}$ & 16 & 32 \\
\hline
\end{tabular}

Table 2: Dihedral f-Tilings of the Sphere by Equilateral and Scalene Triangles

\section{References}

[1] C.P. Avelino and A. F. Santos, Spherical f-Tilings by Triangles and $r$-Sided Regular Polygons, $r \geq 5$, Electron. J. Combin., 15 (2008), \#R22.

[2] A. M. d'Azevedo Breda, A Class of Tilings of $S^{2}$, Geom. Dedicata, 44 (1992), 241253.

[3] A. M. d'Azevedo Breda, P. S. Ribeiro and A. F. Santos, A Class of Spherical Dihedral $f$-Tilings, accepted for publication in European J. Combin.

[4] A. M. d'Azevedo Breda, P. S. Ribeiro and A. F. Santos, Dihedralf-tilings of the Sphere by Equilateral and Scalene Triangles-I, submitted for publication.

[5] A. M. d'Azevedo Breda, P.S. Ribeiro and A.F. Santos, Dihedral f-Tilings of the Sphere by Equilateral and Scalene Triangles-II, Electron. J. Combin., 15 (2008), R91.

[6] A. M. d'Azevedo Breda and A. F. Santos, Dihedral F-Tilings of the Sphere by Spherical Triangles and Equiangular Well Centered Quadrangles, Beiträge Algebra Geometrie, 45 (2004), 441-461.

[7] A. M. d'Azevedo Breda and A. F. Santos, Dihedral f-Tilings of the Sphere by Rhombi and Triangles, Discrete Math. Theor. Comput. Sci., 7 (2005), 123-140.

[8] R. J. Dawson, Tilings of the Sphere with Isosceles Triangles, Discrete Comput. Geom., 30 (2003), 467-487.

[9] R. J. Dawson and B. Doyle, Tilings of the Sphere with Right Triangles I: the Asymptotically Right Families, Electron. J. Combin., 13 (2006), R48.

[10] S. A. Robertson, Isometric Folding of Riemannian Manifolds, Proc. Royal Soc. Edinb. Sect. A, 79 (1977), 275-284. 